\title{
Mineralogy and Geochemistry of Nephrite Jade from Yinggelike Deposit, Altyn Tagh (Xinjiang, NW China)
}

\author{
Ying Jiang ${ }^{1}$, Guanghai Shi ${ }^{1} * \mathbb{D}$, Liguo $\mathrm{Xu}^{2}$ and Xinling $\mathrm{Li}^{3}$ \\ 1 State Key Laboratory of Geological Processes and Mineral Resources, China University of Geosciences, \\ Beijing 100083, China; 3009150004@cugb.edu.cn \\ 2 Geological Museum of China, Beijing 100034, China; ytxlgzidane@hotmail.com \\ 3 Xinjiang Uygur Autonomous Region Product Quality Supervision and Inspection Institute, \\ Xinjiang 830004, China; lx12326164@163.com \\ * Correspondence: shigh@cugb.edu.cn; Tel.: +86-010-8232-1836
}

Received: 6 April 2020; Accepted: 6 May 2020; Published: 8 May 2020

\begin{abstract}
The historic Yinggelike nephrite jade deposit in the Altyn Tagh Mountains (Xinjiang, NW China) is renowned for its gem-quality nephrite with its characteristic light-yellow to greenish-yellow hue. Despite the extraordinary gemological quality and commercial significance of the Yinggelike nephrite, little work has been done on this nephrite deposit, due to its geographic remoteness and inaccessibility. This contribution presents the first systematic mineralogical and geochemical studies on the Yinggelike nephrite deposit. Electron probe microanalysis, X-ray fluorescence (XRF) spectrometry, inductively coupled plasma mass spectrometry (ICP-MS) and isotope ratio mass spectrometry were used to measure the mineralogy, bulk-rock chemistry and stable $(\mathrm{O}$ and $\mathrm{H})$ isotopes characteristics of samples from Yinggelike. Field investigation shows that the Yinggelike nephrite orebody occurs in the dolomitic marble near the intruding granitoids. Petrographic studies and EMPA data indicate that the nephrite is mainly composed of fine-grained tremolite, with accessory pargasite, diopside, epidote, allanite, prehnite, andesine, titanite, zircon, and calcite. Geochemical studies show that all nephrite samples have low bulk-rock Fe/(Fe $+\mathrm{Mg})$ values (0.02-0.05), as well as low $\mathrm{Cr}(0.81-34.68 \mathrm{ppm}), \mathrm{Co}(1.10-2.91 \mathrm{ppm})$, and $\mathrm{Ni}(0.52-20.15 \mathrm{ppm})$ contents. Chondrite-normalized REE patterns of most samples exhibit strong to moderate negative Eu anomalies (0.04-0.67), moderate LREE enrichments, nearly flat HREE patterns, and low $\Sigma$ REE contents $(2.16-11.25 \mathrm{ppm})$. The nephrite samples have $\delta^{18} \mathrm{O}$ and $\delta \mathrm{D}$ values of 5.3 to $7.4 \%$ ond -74.9 to $-86.7 \%$, respectively. The mineralogy, bulk-rock chemistry, and $\mathrm{O}-\mathrm{H}$ isotope characteristics are consistent with the dolomite-related nephrite classification. Based on mineral paragenetic relationships, three possible mineral crystallization stages are recognized: (1) diopside formed by prograde metasomatism; (2) nephrite jade formed by retrograde metasomatism and replacement of Stage I anhydrous minerals; (3) hydrothermal alteration after the nephrite formation. Features of transition metal contents indicate that the color of the Yinggelike nephrite is likely to be controlled by the $\mathrm{Fe}^{2+}, \mathrm{Fe}^{3+}$, and $\mathrm{Mn}$. Yellowish color is related to $\mathrm{Mn}$ and especially $\mathrm{Fe}^{3+}$, while greenish color is related to $\mathrm{Fe}^{2+}$. Our new mineralogical and geochemical results on the Yinggelike nephrite provide better constraints on the formation of other nephrite deposits in the Altyn Tagh Mountains, and can facilitate future nephrite prospecting and research in the region.
\end{abstract}

Keywords: nephrite jade; dolomite-related nephrite; Altyn Tagh Mountains; Xinjiang (NW China); tremolite; mineralogy; geochemistry 


\section{Introduction}

Nephrite is essentially a near-monomineralic rock, normally comprises $>95 \%$ microcrystalline amphibole of the tremolite-actinolite series, and shifts close to tremolite $\left(\mathrm{Ca}_{2}\left(\mathrm{Mg}, \mathrm{Fe}_{5} \mathrm{Si}_{8} \mathrm{O}_{22}(\mathrm{OH})_{2}\right)\right.$ in high-quality nephrite jade [1]. The essential mineralogical feature of nephrite is its microstructure, which is characterized by randomly oriented bundles of felted/twisted fabrics [2]. This key microstructure gives nephrite its extreme toughness and makes it a valuable carving material. Nephrite has been used since prehistoric times and has been treasured for its color and toughness in different cultures throughout the history, especially in Europe and Asia, e.g., in Italy [3], Switzerland [4], Russia [5,6], Bulgaria [7,8], Hungary [9], China [2,10,11], Mongolia [12], Pakistan [13], Taiwan (China), Philippines, Malaysia, Vietnam, Thailand, and Cambodia [14].

Nephrite deposits are widespread around the world, but gem-quality ones are uncommon [15]. Major nephrite deposits are located in China [16-24], Russia [25,26], Canada [27], Australia [1], New Zealand [28-30], USA [1], and South Korea [31]. Nephrite can be divided into serpentinite-related (S-type) and dolomite-related (D-type) types according to its origin [1]. S-type nephrite commonly forms along the contact between serpentinite/peridotite and more silicic rocks [1]. Famous and representative S-type nephrite deposits have been found in British Columbia in Canada [27], East Sayan in Russia [25,26], New Zealand [28-30], Jordanów and the adjacent areas in SW Poland [32,33], Taiwan and Manasi in China [34,35]. D-type nephrite forms along the contact between dolomitic marble and igneous intrusions [1], and is reported in many localities in China [17-22], together with Chuncheon (Korea) [31], Cowell (South Australia) [1], Vitim (Siberia, Russia) [25,26], Złoty Stok (SW Poland) [33,36], and some new discoveries were made in Europe [1]. Previous studies have suggested that S-type and D-type nephrites are of metasomatic and metamorphic origin, respectively [27]. However, recent studies have suggested that D-type nephrite is formed by contact metasomatic replacement of dolomitic marble by Si-rich aqueous fluids during felsic pluton emplacement $[1,18,19,31]$.

In NW China, the giant Hetian nephrite belt extends about $1300 \mathrm{~km}$ from the West Kunlun Mountains to the Altyn Tagh Mountains, forming the largest nephrite belt in the world and hosting tens of important nephrite deposits. Magmatic intrusions in this belt were likely the main driving force behind the nephrite formation [16]. Compared to the many studies on the nephrite deposits in the West Kunlun [17-21], nephrite deposits in the Altyn Tagh have been much less investigated [37-40]. With more nephrite deposits being discovered in the recent years, the Altyn Tagh Mountains have become a major nephrite production region in China, with over half of the nephrites mined in the Qiemo and Ruoqiang Counties [40].

The Yinggelike nephrite deposit (Ruoqiang County) is famous for producing the highly valued yellow-hue nephrite, which is locally called the "Huangkou variety" (i.e., (greenish)-yellow variety). The deposit was first mined in the late Manchurian "Qing" Dynasty (early 1900s), paused for over 100 years due to geopolitical instability, and the production restarted in 2004 [41]. In recent years, the demand for and price of the high-quality yellow nephrite has increased markedly [42]. Despite the extraordinary gemological quality and commercial significance of the Yinggelike nephrite, it has not been well studied due to the geographic remoteness and inaccessibility of the deposit, including tough mountain traffic, difficult terrains, and high elevation. Previous studies only briefly introduced the geological features of the Yinggelike deposit [43,44], but no research has been carried out on the nephrite mineralogy and geochemistry of this deposit. Therefore, this study provides the first systematic geological investigation, mineralogical and geochemical analyses, with the aims of establishing the mineral crystallization stages and the gemological significance of the nephrite.

\section{Geological Background}

\subsection{Hetian Nephrite Belt}

The Hetian nephrite belt is located in the southern part of the Tarim Basin, along the Kunlun and Altyn Tagh Mountains (Figure 1a) [16]. This belt is divided into three geographic sections, 
i.e., (from west to east) Shache-Yecheng $\left(76-78^{\circ} \mathrm{E}\right)$, Hetian-Yutian $\left(78-82^{\circ} \mathrm{E}\right)$, and Qiemo-Ruoqiang $\left(85-90^{\circ}\right.$ E) (Figure 1a) [16]. Recent studies show that this belt contains over 20 nephrite deposits with similar geological setting, mostly located at the contact between dolomitic marble and intermediate-felsic intrusion [19]. The nephrite deposits from the Shache-Yecheng, and Hetian-Yutian sections are distributed in the Kunlun Mountains, while those from Qiemo-Ruoqiang section are distributed in the Altyn Tagh Mountains (Figure 1a).

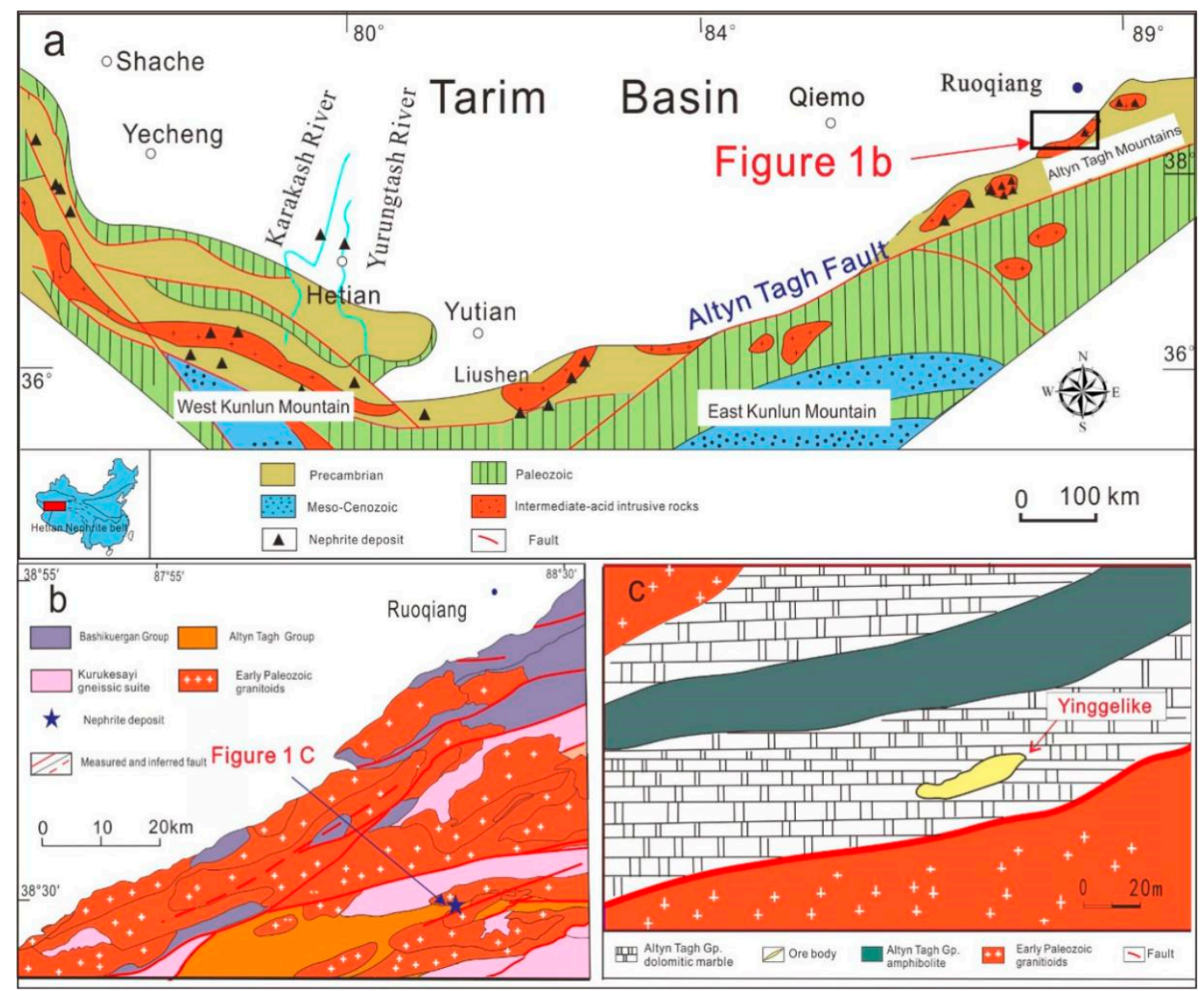

Figure 1. (a) Simplified geologic map of the Hetian nephrite belt, showing the three major sections of Shache-Yecheng $\left(76-78^{\circ}\right.$ E), Hetian-Yutian (78-82 ${ }^{\circ}$ E), and Qiemo-Ruoqiang $\left(85-90^{\circ} \mathrm{E}\right)$ (modified after $[16,18])$; (b) Geologic map of the study area, showing the location of Yinggelike nephrite deposit (modified after [43]); (c) Geologic map of the Yinggelike nephrite orebody (modified after [44]).

\subsection{Qiemo-Ruoqiang Section ( $85-90^{\circ}$ E)}

The Qiemo-Ruoqiang nephrite belt is located in the Altun Tagh Mountains. Altyn Tagh is situated between the Tarim Craton in the north, and the Qaidam block, Qilian and East Kunlun orogens, and the Tibetan plateau in the south [45-49]. Tectonically, the Altyn Tagh is bounded to the north by the south-dipping North Altyn Tagh thrust fault, and to the south by the Altyn Tagh sinistral strike-slip fault [45-49].

The Yinggelike nephrite deposit (Ruoqiang County) is located geologically inside the Central Altyn massif [45]. Local stratigraphy comprises mainly the Bashikuergan Group, the Altyn Tagh Group, and the Kurukesayi gneissic suite (Figure 1b) [43]. The Bashikuergan Group contains weakly metamorphosed clastic sedimentary rocks, interpreted to be Mesoproterozoic. The Paleoproterozoic Altyn Tagh Group is mainly composed of amphibolite, dolomitic marble, schist, gneiss, quartzite and mafic-ultramafic rocks. The Neoproterozoic Kurukesayi gneissic suite is a set of metamorphosed granodiorite-monzogranite-K-feldspar granite. This gneissic suite was intruded and dismembered by 
the locally widespread early Paleozoic granitoids. Major faults in the study area are ENE-trending. This area has likely experienced multiple orogenic processes, accompanied by multiphase and intensive tectono-magmatic activities [43].

The Yinggelike open pit is situated around $70 \mathrm{~km}$ south of Ruoqiang County and is more than $3200 \mathrm{~m}$ above sea-level. The Paleoproterozoic dolomitic marble and amphibolite of Altyn Tagh Group, and early Paleozoic granitoids are exposed in the mine (Figures $1 \mathrm{c}$ and 2a,b). The nephrite is found in the folded grayish-white/-black banded dolomitic marble, and is restricted by faults developed along the contact zone between dolomitic marble and granitoids (Figure 2a,b).
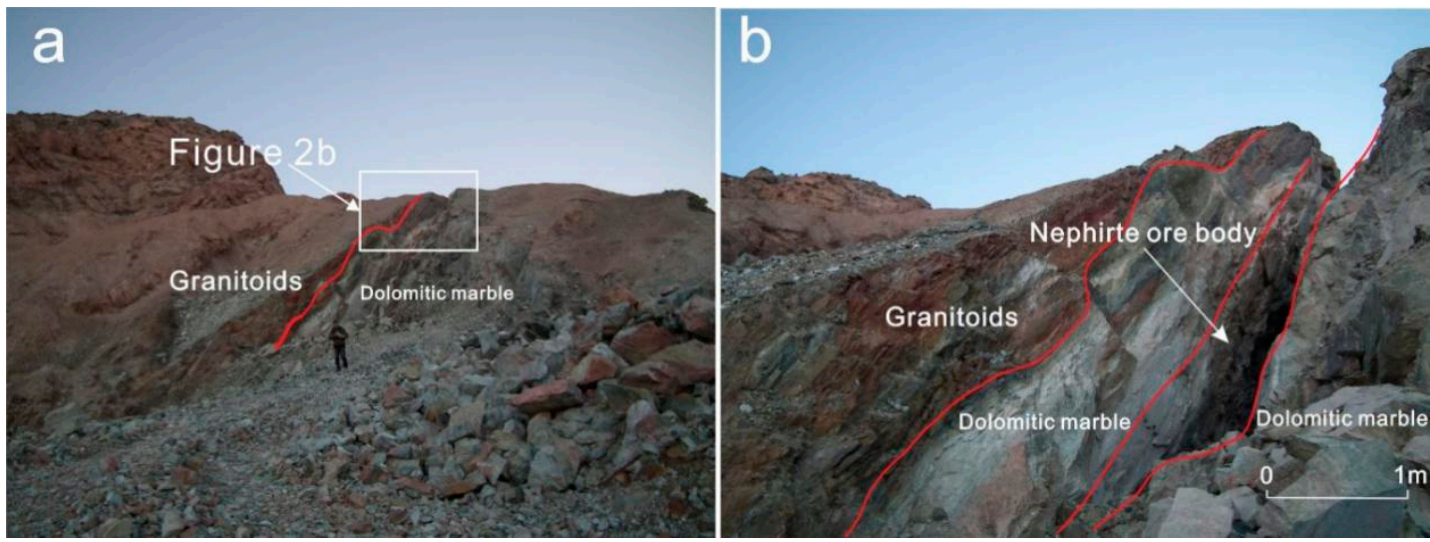

Figure 2. Field photographs of the Yinggelike nephrite mine. (a) Contact between granitoids and dolomitic marble; (b) zoom-in of Figure 2a, showing a nephrite ore vein occurring near the intrusive contact in the dolomitic marble.

\section{Samples and Methods}

\subsection{Samples}

Eleven nephrite samples were collected from the Yinggelike nephrite deposit, of which five

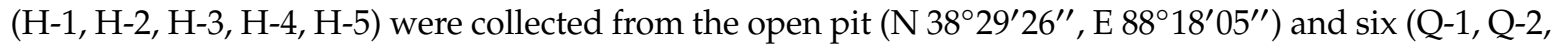
Q-3, Q-4, Q-5, Q-6) were purchased from the mine. Refractive index (RI), specific gravity (SG), and Vickers hardness of all the samples were determined. The RI and SG were examined by the distant vision method and hydrostatic weighing method, respectively, following the procedures outlined by $[50,51]$. The Vickers hardness was examined by the Microvickers hardness tester and converted to Mohs hardness by calculation [52]. During the hardness test, $1 \mathrm{kgf}$ was used as the load value. The gemological properties are listed in Table 1.

Table 1. Gemological properties of the nephrite jade samples from the Yinggelike deposit in Altyn Tagh, NW China.

\begin{tabular}{|c|c|c|c|c|c|c|}
\hline Samples & Color & Luster & Refractive Index & Specific Gravity & Vickers Hardness & Mohs Hardness \\
\hline $\mathrm{H}-1$ & Light-yellow & Sub-vitreous & 1.60 & 2.94 & 638 & 5.8 \\
\hline $\mathrm{H}-2$ & Greenish-yellow & Greasy & 1.60 & 2.88 & 753 & 6.1 \\
\hline $\mathrm{H}-3$ & Yellow & Sub-vitreous & 1.60 & 2.93 & 702 & 6.0 \\
\hline $\mathrm{H}-4$ & Light-yellow & Greasy & 1.60 & 2.96 & 657 & 5.9 \\
\hline H-5 & Light-yellow & Greasy & 1.60 & 2.93 & 784 & 6.2 \\
\hline Q-1 & Greenish-yellow & Sub-vitreous & 1.61 & 2.90 & 713 & 6.0 \\
\hline Q-2 & Greenish-yellow & Sub-vitreous & 1.61 & 2.92 & 733 & 6.1 \\
\hline Q-3 & Greenish-yellow & Sub-vitreous & 1.62 & 2.93 & 738 & 6.1 \\
\hline Q-4 & Light-yellow & Sub-vitreous & 1.62 & 2.96 & 690 & 6.0 \\
\hline Q-5 & Light-yellow & Sub-vitreous & 1.62 & 2.90 & 698 & 6.0 \\
\hline Q-6 & Light-yellow & Sub-vitreous & 1.61 & 2.92 & 750 & 6.1 \\
\hline
\end{tabular}




\subsection{Methods}

Chemical compositions of nephrite and backscattered electron (BSE) images were acquired at the Institute of Geology, Chinese Academy of Geological Sciences (CAGS) in Beijing, China, using a JXA-8100 Electron Microprobe Analyzer (EMPA). Analysis conditions included $15 \mathrm{kV}$ acceleration voltage, $10 \mathrm{nA}$ beam current, and $3 \mu \mathrm{m}$ spot size. The analytical precision was $\pm 2 \%$. Matrix corrections were carried out using the ZAF correction program supplied by the EMPA manufacturer. The EMPA standards included: andradite for $\mathrm{Si}$ and $\mathrm{Ca}$, rutile for $\mathrm{Ti}$, corundum for $\mathrm{Al}$, hematite for $\mathrm{Fe}$, eskolaite for $\mathrm{Cr}$, rhodonite for $\mathrm{Mn}$, bunsenite for $\mathrm{Ni}$, periclase for $\mathrm{Mg}$, albite for $\mathrm{Na}$, $\mathrm{K}$-feldspar for $\mathrm{K}$ and barite for Ba.

Bulk-rock chemical compositions were acquired using an AXIODmAX X-ray fluorescence (XRF) spectrometer at the Hebei Institute of Regional Geology and Mineral Resources (HIRGMR) in Langfang, China. All samples were cleaned, crushed, and milled to $<200$ mesh with an agate mortar. Whole-rock powder samples $(0.7 \mathrm{~g})$ were mixed with $5.2 \mathrm{~g} \mathrm{Li}_{2} \mathrm{~B}_{4} \mathrm{O}_{7}, 0.4 \mathrm{~g} \mathrm{LiF}, 0.3 \mathrm{~g} \mathrm{NH}_{4} \mathrm{NO}_{3}(500 \mathrm{~g} / \mathrm{L})$ in a $25 \mathrm{~mL}$ porcelain crucible. The powder mixture was transferred to a platinum alloy crucible, and $1 \mathrm{~mL}$ $\mathrm{LiBr}$ solution was added before the sample was dried. The powder mixture was then melted in an automatic flame fusion machine at $1200^{\circ} \mathrm{C}$ for $10 \mathrm{~min}$ and casted into glass discs for XRF analysis. Standard samples were prepared using the same procedure. The XRF operating conditions included an accelerating voltage of $30 \mathrm{kV}$ (for Na-P, K, Ca), $40 \mathrm{kV}$ (Ti) and $60 \mathrm{kV}(\mathrm{Mn}, \mathrm{Fe}$ ), beam current of $120 \mathrm{~mA}$ (for Na-P, K, Ca), $90 \mathrm{~mA}$ (Ti) and $60 \mathrm{~mA}(\mathrm{Mn}, \mathrm{Fe}$ ) and collimators of $300 \mu \mathrm{m}$ (for $\mathrm{Al}, \mathrm{Si}$ ) and $700 \mu \mathrm{m}$ (all others). Analytical uncertainties were generally better than $1 \%$. FeO was determined by $\mathrm{K}_{2} \mathrm{Cr}_{2} \mathrm{O}_{7}$ titration after the samples were dissolved in $\mathrm{HF}$ and $\mathrm{H}_{2} \mathrm{SO}_{4}$.

Concentrations of trace elements (including rare earth elements (REEs)) were obtained using an $X$ Series II inductively coupled plasma mass spectrometer (ICP-MS) at HIRGMR. The samples were ground in an agate mortar to 200 mesh. The analysis procedure followed the Silicate Rocks-Part 30as method recommended by the State Standard of the People's Republic of China (GB) [53]. The operating conditions included an analogue detector voltage of $1890 \mathrm{~V}$, PC detector voltage of $2890 \mathrm{~V}$, vacuum level of 2 to 2.4 mbar, scanning time of $60 \mathrm{~s}$, and integration time per element of $0.5 \mathrm{~s}$. The internal standard Rh was used to correct for any analytical drift and matrix effects. The standard deviations for trace elements in the blank samples varied from 0.0005 to 0.8759 and were generally less than 0.05 .

Oxygen and hydrogen isotope analyses were acquired in the laboratory at the Beijing Research Institute of Uranium Geology. Nephrite samples were first crushed to $<60$ mesh to separate the minerals. The rare impurities were removed by hand-picking under the microscope to ensure high purity. The purified samples were then ground to 200 mesh in an agate mortar for oxygen and hydrogen isotope analyses. Oxygen was liberated from the samples by reaction with $\mathrm{BrF}_{5}$ [54] and converted to $\mathrm{CO}_{2}$ on a platinum-coated graphite rod. Oxygen isotope compositions were measured with a Delta V Advantage Isotope Ratio Mass Spectrometer. Analyses of GBW-04409 (quartz) during this study yielded an average $\delta^{18} \mathrm{O}$ value of $11.11 \%$ o (recommended value $=11.11 \%$; Chinese Standard Reference Material GBW-04409) [55]. Precision of the oxygen isotope analyses is better than $0.2 \%$. For hydrogen isotopic compositions, the samples were first degassed of labile volatiles by heating under vacuum to $120^{\circ} \mathrm{C}$ for $3 \mathrm{~h}$. Constitutional water was released by heating the samples to approximately $1200^{\circ} \mathrm{C}$ in an induction furnace. The released water was converted to hydrogen by passage over heated zinc powder at $400{ }^{\circ} \mathrm{C}$ [56]. The hydrogen isotope compositions were analyzed using a MAT-253 Mass Spectrometer with $\pm 2 \%$ o precision. Analyses of Peking University standard water during this study gave an average $\delta \mathrm{D}$ value of $-64 \%$ o (recommended value $=-64.8 \%$; Chinese Standard Reference Material PKU Standard Water) [55]. Stable isotope data for hydrogen and oxygen are reported as $\delta \mathrm{D}$ and $\delta^{18} \mathrm{O}$ per mille (\%) relative to SMOW.

Samples H-1 H-5 were cut into small slabs, and polished thin sections were prepared for petrographic observations. Samples Q-1 Q-6 were cut to uniform-sized $\left(5 \times 2 \times 1 \mathrm{~cm}^{3}\right)$ polished slabs to facilitate the color comparison (Figure 4). 


\section{Results}

\subsection{Gemological Properties}

The conventional gemological properties of the 11 Yinggelike nephrite samples are listed in Table 1. The nephrite samples appear similar to typical nephrites except for the color. All the samples are light-yellow to greenish-yellow with fine texture (Figures 3 and 4), and are translucent with sub-vitreous to greasy luster. Polished samples have commonly sub-vitreous luster. Their reflective index (RI) ranges from 1.60 to 1.62, and specific gravity (SG) from 2.88 to 2.96. The Mohs hardness ranges from 5.8 to 6.2. The gemological properties are similar to typical nephrite jade [23,57].
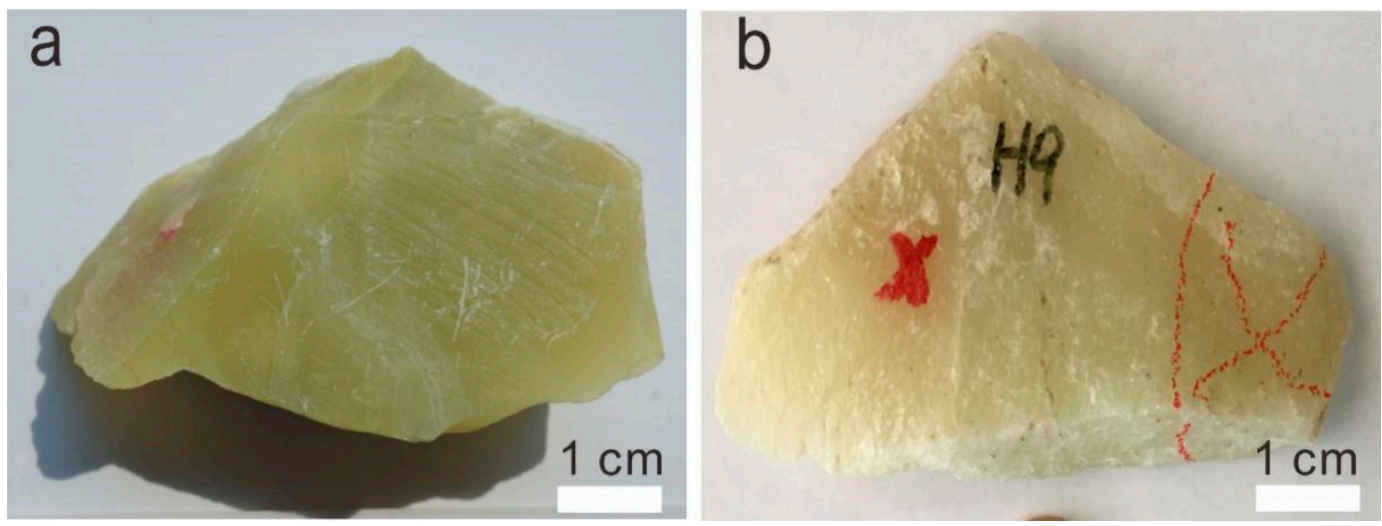

Figure 3. Representative hand specimen photographs of nephrite collected from the Yinggelike open pit. (a) Representative greenish-yellow sample; (b) representative light-yellow sample.

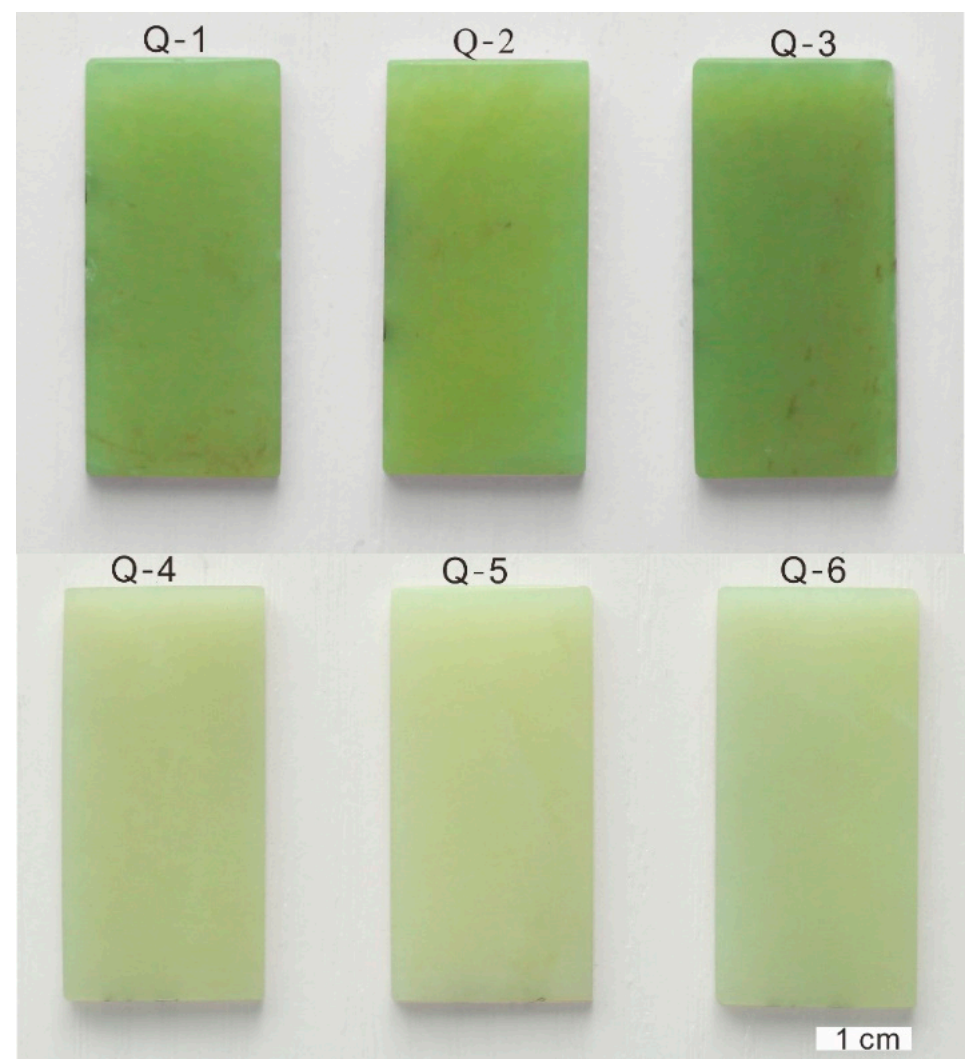

Figure 4. Nephrite samples purchased from the Yinggelike mine. Samples Q-1, Q-2, and Q-3 are greenish-yellow, and Q-4, Q-5, and Q-6 are light-yellow. 


\subsection{Petrography}

All the nephrite samples comprise predominantly tremolite $(\mathrm{Tr} ;>98$ vol.\%), with minor pargasite $(\operatorname{Prg})$, diopside $(\mathrm{Di})$, epidote $(\mathrm{Ep})$, allanite $(\mathrm{Aln})$, andesine (Ads), prehnite (Prh), titanite (Ttn), zircon (Zrn), calcite (Cal), etc. Occurrences of these minerals are described below.

\subsubsection{Tremolite}

Based on the occurrence, paragenesis and chemical composition, two generations of tremolite can be classified: undeformed (Tr-I) and deformed (Tr-II). Tr-I is very rare and occurs as euhedral porphyroblasts of approximately $200 \mu \mathrm{m}$ long and $60 \mu \mathrm{m}$ wide (Figure 5a). Some Tr-I grains form rhombohedral pseudomorphs of carbonates (Figures $5 \mathrm{~b}$ and $6 \mathrm{a}$ ) and were partially replaced by Tr-II. In some cases, Tr-I grains also replaced rounded/oval diopside, and were themselves replaced by Tr-II (Figure 6b). The fine-grained, non-directional micro/cryptocrystalline Tr-II grains are the main form of tremolite in the Yinggelike nephrite (Figure 5). Aggregates of Tr-II commonly exhibit fine fibrous-felted texture (Figure 5c).

\subsubsection{Pargasite}

Pargasite is commonly found in the Yinggelike nephrite. Some pargasite grains appeared as euhedral crystals in the nephrite, with sizes of about $400 \mu \mathrm{m}$ in length and $260 \mu \mathrm{m}$ in width (Figure $5 \mathrm{~d}$ ), while some grains were partially replaced by tremolite (Figure $6 c, d$ ). Pargasite also replaced diopside locally (Figure 6c), indicating that it had formed before tremolite but after diopside. It is noted that this mineral is rare in other nephrite deposits in Hetian nephrite belt $[18,19,37,38]$.

\subsubsection{Diopside}

Diopside grains are generally irregularly shaped (Figure 5e) and relatively common in the Yinggelike nephrite. They were commonly replaced by tremolite along their grain boundary or cracks (Figures 5e and 6b).

\subsubsection{Epidote, Allanite, and Prehnite}

Most epidote and allanite grains are idiomorphic columnar (Figure $5 \mathrm{f}-\mathrm{g}$ ) with local tremolite replacement (Figure 6e,f). Prehnite displays commonly metasomatic relict texture via tremolite replacement (Figure 6g).

\subsubsection{Andesine, Titanite, Zircon, and Calcite}

Andesine, titanite, and zircon are only distributed sporadically in some samples (Figure 6f,h,i). Andesine, which exhibits metasomatic relict texture, was replaced by tremolite. The titanite and zircon are relatively euhedral, and were formed before tremolite. Late-stage calcite veins intruded the Tr-II fractures locally (Figure 5 h). 

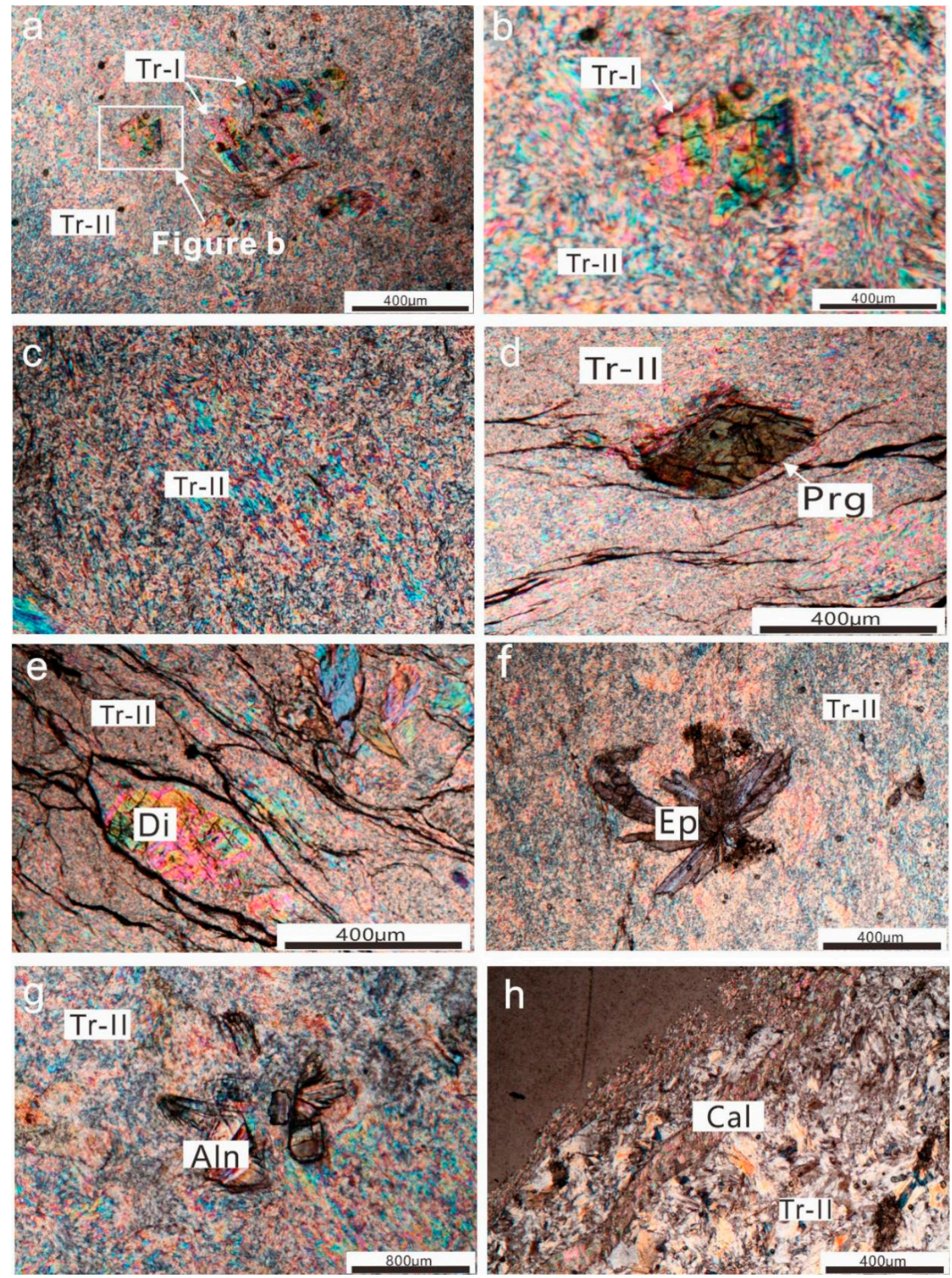

Figure 5. Photomicrographs of the Yinggelike nephrite (crossed-polarized light). The nephrite groundmass comprises mainly fine-fibrous Tr-II. (a) Coarse-grained Tr-I in nephrite (H-2); (b) Tr-I grains in carbonate pseudomorph (H-2), zoom-in of Figure 5a; (c) Fine-grained Tr-II (H-1); (d) Single pargasite crystal in nephrite (H-4); (e) Replacement of diopside by fine-fibrous Tr-II (H-3); (f,g) Idiomorphic epidote (H-3) and allanite (H-5) grains in nephrite; (h) Late-stage calcite vein in nephrite (H-3). 

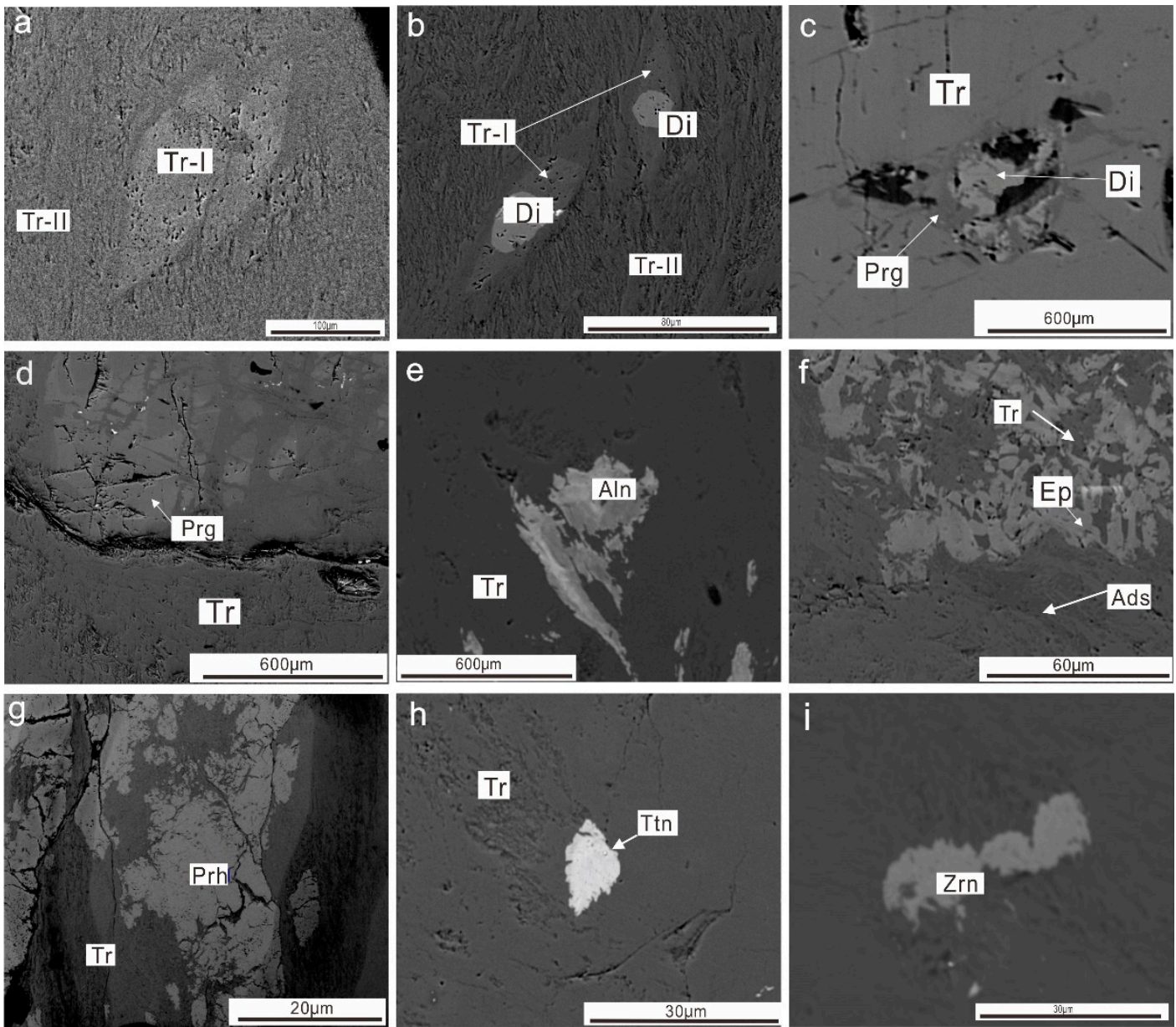

Figure 6. Back-scattered electron (BSE) images of the Yinggelike nephrite. (a) Tremolite grains in carbonate pseudomorph (H-2); (b) Rounded/oval-shaped diopside replaced by Tr-I, which was in turn replaced by Tr-II (H-5); (c) Diopside replaced by pargasite, which was in turn replaced by tremolite (H-5); (d) Replacement of pargasite by tremolite (H-5); (e-g) Metasomatic relicts of allanite (H-5), epidote, andesine and prehnite (H-3); (h) Idiomorphic titanite (H-1); (i) Irregularly shaped zircon crystal (H-5).

\subsection{Mineral Chemistry}

\subsubsection{Amphibole}

According to the amphibole classification diagram (Figure 7), all the Yinggelike amphiboles belong to the calcic group, and are classified as tremolite and pargasite [58]. Tremolite is mainly composed of (in wt.\%) $\mathrm{SiO}_{2}$ (56.96-59.74), $\mathrm{CaO}$ (12.54-13.89), $\mathrm{MgO}$ (21.05-24.62), total $\mathrm{Fe}$ as $\mathrm{FeO}$ $\left(\mathrm{FeO}_{\mathrm{T}}=0.27-3.48\right)$, with atoms per formula unit (a.p.f.u.) $\mathrm{Si}=7.88-8.00$, $\mathrm{Ca}$ on $\mathrm{B}$ site $>1.50$, $(\mathrm{Na}+\mathrm{K})$ on A site $<0.5$ (Table 2). Their $\mathrm{Mg} /\left(\mathrm{Mg}+\mathrm{Fe}^{2+}\right)$ ratios vary from 0.92 to $1.00 . \mathrm{Tr}$-I grains have higher $\mathrm{FeO}_{\mathrm{T}}$ content than that of Tr-II. Pargasite is composed of (in wt.\%) $\mathrm{SiO}_{2}$ (44.90-45.90), $\mathrm{CaO}$ (13.96-14.50), $\mathrm{MgO}$ (18.99-19.76), $\mathrm{FeO}_{\mathrm{T}}(0.23-0.52)$. The pargasite grains are featured with $\mathrm{Si}=6.29-6.39$ a.p.f.u., $\mathrm{Ca}$ on $\mathrm{B}$ site $>1.50,(\mathrm{Na}+\mathrm{K})$ on A site $>0.5$, and $\mathrm{Mg} /\left(\mathrm{Mg}+\mathrm{Fe}^{2+}\right)=0.99-1.00$ (Table 2). $\mathrm{FeO}_{\mathrm{T}}$ contents in $\mathrm{Tr}-\mathrm{II}$ and pargasite are low. The calculation of $\mathrm{Fe}^{3+}$ in amphibole is based on the average estimation method of Schumacher [58].

\subsubsection{Diopside}

Diopside grains are mainly composed of (in wt.\%) $\mathrm{SiO}_{2}$ (54.20-55.03), $\mathrm{CaO}$ (25.93-26.94), $\mathrm{MgO}$ (16.50-18.47), $\mathrm{FeO}_{\mathrm{T}}(0.05-0.40)$, with (a.p.f.u.) $\mathrm{Si}=1.96-1.98, \mathrm{Mg}=0.89-0.99$, and $\mathrm{Ca}=1.00-1.04$ 
(Table 3). It is almost homogeneous, and compositionally is close to the diopside end-member [59]. The $\mathrm{FeO}$ and $\mathrm{Fe}_{2} \mathrm{O}_{3}$ contents were calculated by charge balance method.

\subsubsection{Epidote, Allanite, Andesine, Prehnite, Titanite, Zircon, and Calcite}

The chemical compositions of other minor minerals are presented in Table A1. Epidote is mainly composed of (in wt.\%) $\mathrm{SiO}_{2}$ (37.14-37.75), $\mathrm{CaO}$ (25.71-25.72), $\mathrm{Al}_{2} \mathrm{O}_{3}$ (28.05-28.30), $\mathrm{FeO}_{\mathrm{T}}$ (3.40-3.78). Both allanite and epidote belong to the epidote group. Different from epidote, the allanite contains considerable REE contents (10.89-12.18), as well as $\mathrm{SiO}_{2}$ (35.64-36.25), $\mathrm{CaO}$ (18.14-20.11), $\mathrm{Al}_{2} \mathrm{O}_{3}$ (23.22-24.73), $\mathrm{FeO}_{\mathrm{T}}$ (5.11-7.24). One andesine grain contains (in wt.\%) $\mathrm{SiO}_{2}$ (61.19), $\mathrm{Al}_{2} \mathrm{O}_{3}(24.32$ ), $\mathrm{CaO}(7.27)$, and $\mathrm{Na}_{2} \mathrm{O}(7.23) . \mathrm{Ca} /(\mathrm{Ca}+\mathrm{Na})$ ratio is 0.36 . The prehnite grains contain (in wt.\%): $\mathrm{SiO}_{2}$ (44.75-44.85), $\mathrm{CaO}(27.01-27.23), \mathrm{Al}_{2} \mathrm{O}_{3}$ (22.86-23.53), and $\mathrm{FeO}_{\mathrm{T}}(0.17-0.22)$.

Table 2. Chemical compositions of tremolite (Tr-I, Tr-II) in the Yinggelike nephrite (wt.\%).

\begin{tabular}{|c|c|c|c|c|c|c|c|c|c|c|c|c|c|}
\hline Samples & H-1 & H-2 & H-3 & H-4 & H-5 & Q-1 & Q-2 & Q-3 & Q-4 & Q-5 & Q-6 & H-2 & H-5 \\
\hline Minerals & Tr-II & Tr-II & Tr-II & Tr-II & Tr-II & Tr-II & Tr-II & Tr-II & Tr-II & Tr-II & Tr-II & Tr-I & Tr-I \\
\hline $\mathrm{SiO}_{2}$ & 58.34 & 58.77 & 58.31 & 59.74 & 59.35 & 59.21 & 59.09 & 57.90 & 58.06 & 58.62 & 58.40 & 56.96 & 57.07 \\
\hline $\mathrm{TiO}_{2}$ & 0.00 & 0.00 & 0.01 & 0.01 & 0.00 & 0.02 & 0.00 & 0.00 & 0.02 & 0.04 & 0.03 & 0.00 & 0.00 \\
\hline $\mathrm{Al}_{2} \mathrm{O}_{3}$ & 0.76 & 0.72 & 0.78 & 0.60 & 0.63 & 0.63 & 0.72 & 0.69 & 0.72 & 1.62 & 0.76 & 1.08 & 2.02 \\
\hline $\mathrm{FeO}$ & 0.97 & 0.98 & 0.94 & 0.43 & 0.37 & 0.35 & 0.27 & 0.45 & 1.96 & 1.13 & 1.06 & 3.48 & 2.66 \\
\hline $\mathrm{Cr}_{2} \mathrm{O}_{3}$ & 0.00 & 0.00 & 0.01 & 0.00 & 0.00 & 0.00 & 0.06 & 0.00 & 0.00 & 0.02 & 0.01 & 0.02 & 0.00 \\
\hline $\mathrm{MnO}$ & 0.09 & 0.08 & 0.11 & 0.01 & 0.03 & 0.01 & 0.00 & 0.03 & 0.00 & 0.02 & 0.10 & 0.50 & 0.84 \\
\hline $\mathrm{MgO}$ & 24.09 & 23.75 & 23.95 & 23.90 & 23.19 & 24.62 & 24.17 & 24.07 & 23.42 & 22.69 & 23.94 & 21.63 & 21.05 \\
\hline $\mathrm{CaO}$ & 12.93 & 12.82 & 13.02 & 12.89 & 13.59 & 12.77 & 12.54 & 13.89 & 13.36 & 13.54 & 13.09 & 13.78 & 12.88 \\
\hline $\mathrm{Na}_{2} \mathrm{O}$ & 0.09 & 0.10 & 0.12 & 0.09 & 0.10 & 0.13 & 0.33 & 0.09 & 0.10 & 0.21 & 0.13 & 0.12 & 0.31 \\
\hline $\mathrm{K}_{2} \mathrm{O}$ & 0.14 & 0.08 & 0.12 & 0.08 & 0.05 & 0.11 & 0.20 & 0.07 & 0.08 & 0.06 & 0.15 & 0.08 & 0.14 \\
\hline $\mathrm{NiO}$ & 0.00 & 0.03 & 0.01 & 0.00 & 0.01 & 0.02 & 0.00 & 0.00 & 0.00 & 0.00 & 0.00 & 0.03 & 0.00 \\
\hline Total & 97.42 & 97.33 & 97.39 & 97.75 & 97.31 & 97.86 & 97.38 & 97.19 & 97.72 & 97.93 & 97.67 & 97.68 & 96.97 \\
\hline TSi & 7.96 & 8.00 & 7.96 & 8.00 & 8.00 & 8.00 & 8.00 & 7.91 & 7.93 & 8.00 & 7.95 & 7.88 & 7.94 \\
\hline TAl & 0.04 & 0.00 & 0.04 & 0.00 & 0.00 & 0.00 & 0.00 & 0.09 & 0.07 & 0.00 & 0.05 & 0.12 & 0.07 \\
\hline SumT & 8.00 & 8.00 & 8.00 & 8.00 & 8.00 & 8.00 & 8.00 & 8.00 & 8.00 & 8.00 & 8.00 & 8.00 & 8.00 \\
\hline $\mathrm{CAl}$ & 0.08 & 0.12 & 0.08 & 0.10 & 0.10 & 0.10 & 0.11 & 0.03 & 0.05 & 0.26 & 0.08 & 0.06 & 0.27 \\
\hline $\mathrm{CFe}^{3+}$ & 0.00 & 0.00 & 0.00 & 0.05 & 0.04 & 0.00 & 0.03 & 0.00 & 0.00 & 0.00 & 0.00 & 0.00 & 0.00 \\
\hline $\mathrm{CMg}$ & 4.90 & 4.82 & 4.87 & 4.77 & 4.66 & 4.90 & 4.85 & 4.91 & 4.77 & 4.61 & 4.86 & 4.46 & 4.36 \\
\hline $\mathrm{CFe}^{2+}$ & 0.02 & 0.07 & 0.04 & 0.00 & 0.00 & 0.00 & 0.00 & 0.05 & 0.18 & 0.12 & 0.06 & 0.40 & 0.31 \\
\hline CMn & 0.00 & 0.00 & 0.00 & 0.00 & 0.00 & 0.00 & 0.00 & 0.00 & 0.00 & 0.00 & 0.00 & 0.06 & 0.06 \\
\hline SumC & 5.00 & 5.00 & 5.00 & 4.92 & 4.81 & 5.00 & 5.00 & 4.98 & 5.00 & 5.00 & 5.00 & 4.98 & 5.00 \\
\hline $\mathrm{BMg}$ & 0.00 & 0.00 & 0.00 & 0.00 & 0.00 & 0.06 & 0.03 & 0.00 & 0.00 & 0.00 & 0.00 & 0.00 & 0.00 \\
\hline $\mathrm{BFe}_{2}$ & 0.09 & 0.05 & 0.07 & 0.00 & 0.00 & 0.04 & 0.00 & 0.00 & 0.00 & 0.00 & 0.06 & 0.00 & 0.00 \\
\hline BMn & 0.01 & 0.01 & 0.01 & 0.00 & 0.00 & 0.00 & 0.00 & 0.00 & 0.00 & 0.00 & 0.11 & 0.00 & 0.04 \\
\hline $\mathrm{BCa}$ & 1.89 & 1.87 & 1.90 & 1.85 & 1.96 & 1.85 & 1.82 & 2.00 & 1.96 & 1.98 & 1.91 & 2.00 & 1.92 \\
\hline $\mathrm{BNa}$ & 0.12 & 0.04 & 0.02 & 0.02 & 0.03 & 0.03 & 0.09 & 0.09 & 0.09 & 0.02 & 0.02 & 0.03 & 0.04 \\
\hline SumB & 2.00 & 1.95 & 2.00 & 1.87 & 1.99 & 1.98 & 1.94 & 2.09 & 2.05 & 2.00 & 2.00 & 2.03 & 2.00 \\
\hline $\mathrm{ACa}$ & 0.00 & 0.00 & 0.00 & 0.00 & 0.00 & 0.00 & 0.00 & 0.04 & 0.00 & 0.00 & 0.00 & 0.04 & 0.00 \\
\hline $\mathrm{ANa}$ & 0.12 & 0.00 & 0.02 & 0.00 & 0.00 & 0.00 & 0.00 & 0.02 & 0.03 & 0.04 & 0.02 & 0.03 & 0.04 \\
\hline $\mathrm{AK}$ & 0.03 & 0.01 & 0.02 & 0.01 & 0.01 & 0.02 & 0.04 & 0.01 & 0.01 & 0.01 & 0.03 & 0.01 & 0.03 \\
\hline SumA & 0.15 & 0.01 & 0.04 & 0.01 & 0.01 & 0.02 & 0.04 & 0.07 & 0.04 & 0.05 & 0.05 & 0.09 & 0.07 \\
\hline Sumcat & 15.15 & 14.96 & 15.04 & 14.80 & 14.81 & 15.00 & 14.98 & 15.14 & 15.09 & 15.05 & 15.05 & 15.10 & 15.07 \\
\hline $\mathrm{Mg}^{2+} /\left(\mathrm{Mg}^{2+}+\mathrm{Fe}^{2+}\right)$ & 0.98 & 0.98 & 0.98 & 1.00 & 1.00 & 1.00 & 1.00 & 0.99 & 1.00 & 0.98 & 0.98 & 0.92 & 0.93 \\
\hline
\end{tabular}


Table 3. Chemical compositions of pargasite and diopside in the Yinggelike nephrite (wt.\%).

\begin{tabular}{|c|c|c|c|c|c|c|c|c|}
\hline Samples & H-3 & H-3 & H-4 & H-5 & H-1 & H-5 & H-2 & H-3 \\
\hline Minerals & Prg & Prg & Prg & Prg & Di & Di & Di & Di \\
\hline $\mathrm{SiO}_{2}$ & 45.78 & 45.90 & 44.90 & 45.02 & 55.03 & 54.20 & 54.54 & 54.69 \\
\hline $\mathrm{TiO}_{2}$ & 0.33 & 0.62 & 1.42 & 1.43 & 0.01 & 0.18 & 0.00 & 0.04 \\
\hline $\mathrm{Al}_{2} \mathrm{O}_{3}$ & 14.60 & 15.10 & 14.52 & 14.24 & 0.50 & 0.79 & 0.49 & 2.01 \\
\hline $\mathrm{FeO}$ & 0.52 & 0.50 & 0.52 & 0.23 & 0.12 & 0.07 & 0.05 & 0.40 \\
\hline $\mathrm{Cr}_{2} \mathrm{O}_{3}$ & 0.00 & 0.03 & 0.00 & 0.00 & 0.04 & 0.00 & 0.01 & 0.03 \\
\hline $\mathrm{MnO}$ & 0.03 & 0.00 & 0.00 & 0.04 & 0.00 & 0.04 & 0.04 & 0.07 \\
\hline $\mathrm{MgO}$ & 19.36 & 18.99 & 19.39 & 19.76 & 18.46 & 17.68 & 18.47 & 16.50 \\
\hline $\mathrm{CaO}$ & 14.50 & 14.37 & 13.96 & 14.38 & 26.13 & 26.94 & 26.23 & 25.93 \\
\hline $\mathrm{Na}_{2} \mathrm{O}$ & 2.25 & 1.95 & 1.97 & 1.93 & 0.02 & 0.05 & 0.00 & 0.20 \\
\hline $\mathrm{K}_{2} \mathrm{O}$ & 0.11 & 0.15 & 0.24 & 0.28 & 0.00 & 0.01 & 0.00 & 0.03 \\
\hline $\mathrm{NiO}$ & 0.00 & 0.00 & 0.00 & 0.00 & 0.02 & 0.00 & 0.03 & 0.00 \\
\hline Total & 97.44 & 97.58 & 97.27 & 97.30 & 100.33 & 99.96 & 99.86 & 99.90 \\
\hline Si & 6.39 & 6.39 & 6.29 & 6.29 & 1.98 & 1.96 & 1.97 & 1.98 \\
\hline $\mathrm{Ti}$ & 0.03 & 0.07 & 0.15 & 0.15 & 0.00 & 0.00 & 0.00 & 0.00 \\
\hline $\mathrm{Cr}$ & 0.00 & 0.00 & 0.00 & 0.00 & 0.00 & 0.00 & 0.00 & 0.00 \\
\hline $\mathrm{Al}$ & 2.40 & 2.48 & 2.40 & 2.34 & 0.02 & 0.03 & 0.02 & 0.09 \\
\hline $\mathrm{Fe}^{3+}$ & 0.00 & 0.00 & 0.03 & 0.03 & 0.00 & 0.00 & 0.00 & 0.00 \\
\hline $\mathrm{Mg}$ & 4.03 & 3.94 & 4.05 & 4.12 & 0.99 & 0.95 & 0.99 & 0.89 \\
\hline $\mathrm{Fe}^{2+}$ & 0.06 & 0.06 & 0.03 & 0.00 & 0.00 & 0.00 & 0.00 & 0.01 \\
\hline $\mathrm{Mn}$ & 0.00 & 0.00 & 0.00 & 0.00 & 0.00 & 0.00 & 0.00 & 0.00 \\
\hline $\mathrm{Ca}$ & 2.17 & 2.14 & 2.10 & 2.15 & 1.01 & 1.04 & 1.01 & 1.00 \\
\hline $\mathrm{Na}$ & 0.64 & 0.55 & 0.56 & 0.55 & 0.00 & 0.00 & 0.00 & 0.01 \\
\hline K & 0.02 & 0.03 & 0.04 & 0.05 & 0.00 & 0.00 & 0.00 & 0.00 \\
\hline Sum & 15.75 & 15.66 & 15.66 & 15.68 & 4.00 & 4.00 & 4.00 & 3.99 \\
\hline $\mathrm{BCa}$ & 2.00 & 2.00 & 2.00 & 2.00 & n.d. & n.d. & n.d. & n.d. \\
\hline$(\mathrm{Na}+\mathrm{K})_{\mathrm{A}}$ & 0.57 & 0.64 & 0.58 & 0.57 & n.d. & n.d. & n.d. & n.d. \\
\hline $\mathrm{Mg}^{2+} /\left(\mathrm{Mg}^{2+}+\mathrm{Fe}^{2+}\right)$ & 0.99 & 0.99 & 0.99 & 1.00 & n.d. & n.d. & n.d. & n.d. \\
\hline
\end{tabular}

Amphibole formulae recalculated on the basis of 23 oxygens; 0.00 - concentration below the detection limit; n.d.- not detected. Diopside formulae recalculated on the basis of 4 cations.
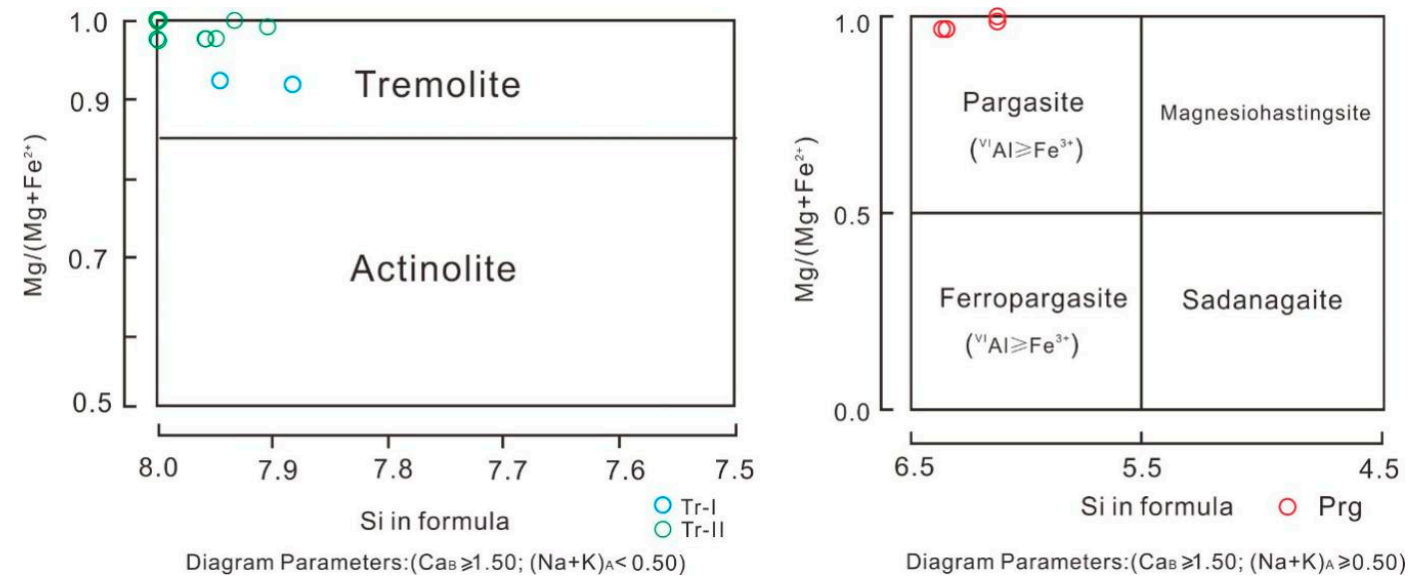

Figure 7. Amphibole classification diagram for the Yinggelike nephrite.

\subsection{Bulk-Rock Chemistry}

All the nephrite samples have narrow ranges of major element contents (in wt.\%): $\mathrm{SiO}_{2}$ (55.05-58.27), $\mathrm{MgO}$ (23.80-27.85), $\mathrm{CaO}$ (11.43-13.21), $\mathrm{TFe}_{2} \mathrm{O}_{3}$ (0.37-1.15), $\mathrm{Al}_{2} \mathrm{O}_{3}$ (0.76-2.64), and low $\mathrm{TiO}_{2}(0.01-0.03)$ and $\mathrm{MnO}(0.02-0.08)$ (Table 4), which resemble theoretical tremolite end-member composition. This is in agreement with our petrographic observations that tremolite is the main component in the nephrite. The $\mathrm{Fe} /(\mathrm{Fe}+\mathrm{Mg})$ ratios are 0.02 to 0.05 . The transition metal contents of the nephrite purchased from the mine are listed in Table 5. As seen in Table 5, $\mathrm{Fe}^{2+}, \mathrm{Fe}^{3+}$ and $\mathrm{Mn}$ contents are generally high in all the samples, followed by $\mathrm{Ti}$, whereas the $\mathrm{V}, \mathrm{Cr}, \mathrm{Co}, \mathrm{Ni}, \mathrm{Cu}$ contents are too low (generally $<10 \mathrm{ppm}$ ) to have significant impact on the color. Therefore, the $\mathrm{Fe}^{2+}, \mathrm{Fe}^{3+}, \mathrm{Mn}$, and Ti may play an important role in Yinggelike nephrite color. 
Table 4. Bulk-rock chemical compositions of the Yinggelike nephrite samples.

\begin{tabular}{ccccccccccccc}
\hline Samples & $\mathbf{H - 1}$ & $\mathbf{H - 2}$ & $\mathbf{H - 3}$ & $\mathbf{H - 4}$ & $\mathbf{H - 5}$ & $\mathbf{Q - 1}$ & $\mathbf{Q - 2}$ & $\mathbf{Q - 3}$ & $\mathbf{Q - 4}$ & $\mathbf{Q - 5}$ & $\mathbf{Q - 6}$ & AVG \\
\hline $\mathrm{SiO}_{2}$ & 55.93 & 55.05 & 55.21 & 56.20 & 56.12 & 57.97 & 57.88 & 58.27 & 57.18 & 55.89 & 57.67 & 56.70 \\
$\mathrm{TiO}_{2}$ & 0.02 & 0.02 & 0.02 & 0.03 & 0.03 & 0.02 & 0.02 & 0.01 & 0.01 & 0.01 & 0.01 & 0.02 \\
$\mathrm{Al}_{2} \mathrm{O}_{3}$ & 0.76 & 1.10 & 2.11 & 2.19 & 0.85 & 0.77 & 1.09 & 0.76 & 1.30 & 2.64 & 1.05 & 1.33 \\
$\mathrm{TFe}_{2} \mathrm{O}_{3}$ & 0.56 & 1.15 & 0.50 & 0.78 & 0.37 & 0.78 & 0.66 & 0.74 & 0.60 & 0.48 & 0.57 & 0.65 \\
$\mathrm{FeO} \mathrm{O}_{3}$ & 0.40 & 0.83 & 0.33 & 0.49 & 0.26 & 0.61 & 0.49 & 0.58 & 0.53 & 0.41 & 0.50 & 0.49 \\
$\mathrm{Fe}_{2} \mathrm{O}_{3}$ & 0.09 & 0.23 & 0.13 & 0.24 & 0.08 & 0.10 & 0.11 & 0.10 & 0.01 & 0.03 & 0.01 & 0.10 \\
$\mathrm{MnO}$ & 0.02 & 0.08 & 0.03 & 0.02 & 0.04 & 0.07 & 0.06 & 0.08 & 0.04 & 0.03 & 0.05 & 0.05 \\
$\mathrm{MgO}$ & 27.44 & 26.49 & 27.44 & 26.88 & 27.85 & 24.25 & 23.81 & 23.80 & 24.51 & 25.07 & 24.63 & 25.70 \\
$\mathrm{CaO}$ & 12.26 & 12.40 & 11.43 & 13.12 & 11.76 & 12.58 & 13.21 & 12.99 & 12.37 & 11.93 & 12.76 & 12.4 \\
$\mathrm{Na} 2$ & 0.12 & 0.09 & 0.15 & 0.13 & 0.14 & 0.44 & 0.39 & 0.47 & 0.41 & 0.36 & 0.33 & 0.28 \\
$\mathrm{~K}_{2} \mathrm{O}$ & 0.08 & 0.09 & 0.22 & 0.14 & 0.07 & 0.20 & 0.11 & 0.11 & 0.61 & 0.39 & 0.41 & 0.22 \\
$\mathrm{P}_{2} \mathrm{O}_{5}$ & 0.01 & 0.01 & 0.01 & 0.03 & 0.01 & 0.01 & 0.02 & 0.01 & 0.01 & 0.01 & 0.01 & 0.01 \\
$\mathrm{LOI}$ & 2.81 & 3.55 & 2.83 & 0.44 & 2.73 & 2.97 & 2.78 & 2.80 & 2.99 & 3.23 & 2.55 & 2.70 \\
$\mathrm{Total}$ & 100.49 & 101.1 & 100.5 & 100.85 & 100.37 & 99.99 & 99.98 & 99.98 & 99.98 & 99.99 & 99.99 & 100 \\
$\mathrm{Fe} /(\mathrm{Fe}+\mathrm{Mg})$ & 0.02 & 0.05 & 0.02 & 0.03 & 0.02 & 0.03 & 0.03 & 0.03 & 0.03 & 0.02 & 0.03 & 0.03 \\
\hline
\end{tabular}

T-Total; LOI-Loss on ignition.

Table 5. Transition metal concentrations (ppm) of the Yinggelike nephrite.

\begin{tabular}{ccccccccccc}
\hline Samples & $\mathbf{F e}^{\mathbf{3 +}}$ & $\mathbf{F e}^{2+}$ & $\mathbf{M n}$ & $\mathbf{T i}$ & $\mathbf{V}$ & $\mathbf{C r}$ & $\mathbf{C o}$ & $\mathbf{N i}$ & $\mathbf{C u}$ & Color \\
\hline Q-1 & 695 & 4731 & 550 & 108 & 11.6 & 4.42 & 1.82 & 3.44 & 0.52 & Greenish-yellow \\
Q-2 & 769 & 3824 & 459 & 118 & 7.42 & 8.81 & 1.87 & 3.51 & 1.04 & Greenish-yellow \\
Q-3 & 733 & 4472 & 578 & 81 & 11.5 & 9.14 & 2.14 & 3.72 & 0.93 & Greenish-yellow \\
Q-4 & 96 & 4083 & 341 & 61 & 1.37 & 8.44 & 1.55 & 4.12 & 1.74 & Light-yellow \\
Q-5 & 184 & 3175 & 210 & 79 & 2.77 & 2.03 & 0.83 & 3.19 & 0.37 & Light-yellow \\
Q-6 & 98 & 3889 & 361 & 88 & 1.49 & 6.03 & 1.56 & 3.46 & 0.41 & Light-yellow \\
\hline
\end{tabular}

$\mathrm{Fe}^{3+}$ and $\mathrm{Fe}^{2+}$ contents were re-calculated (in ppm) from the XRF data; the remaining elements were analyzed by inductively coupled plasma-mass spectrometry (ICP-MS).

The nephrite samples have low total REE contents ( 2.16 to $11.25 \mathrm{ppm}$ ), except for sample H-5 (736.23 ppm) (Table A2; Figure 8). Photomicrographs (Figure 5g), BSE images (Figure 6e,i) and EMPA data (Table A1) suggest that mineral inclusions such as allanite and zircon in sample H-5 have generated higher trace element (including REE) contents than in the other samples (Table A2). Chondrite-normalized REE patterns of the Yinggelike nephrite are characterized by steep LREE and flat HREE patterns (Figure 8) with LREE/HREE of 5.16 to 9.05, and distinct to moderately negative Eu anomalies $\left(\mathrm{Eu} / \mathrm{Eu}^{*}=0.04-0.67\right)$. The nephrite samples have $(\mathrm{La} / \mathrm{Sm})_{\mathrm{N}}=1.72$ to 4.04 and $(\mathrm{Gd} / \mathrm{Yb})_{\mathrm{N}}=0.53$ to 2.62 (Table A2).

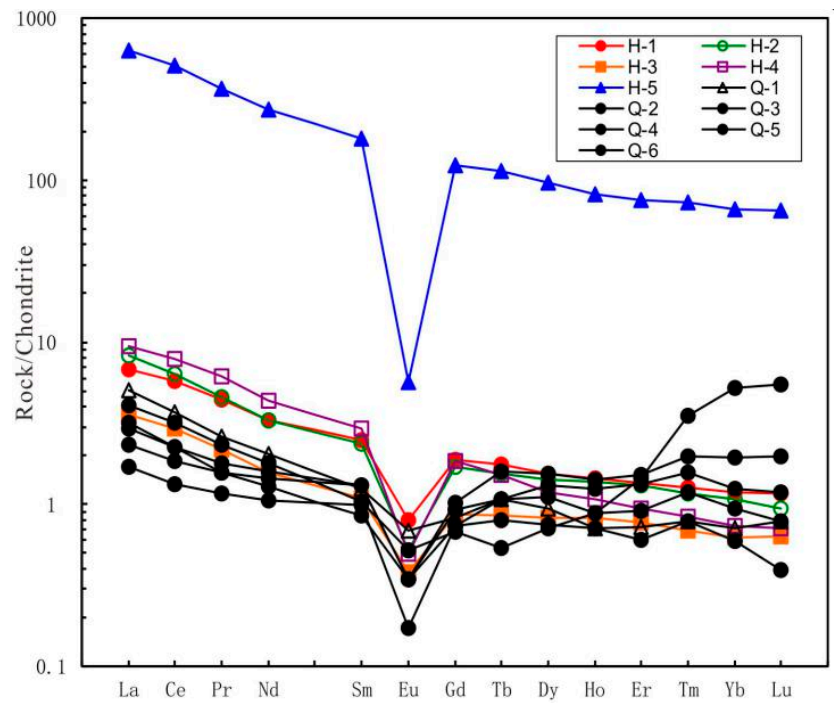

Figure 8. Chondrite-normalized REE patterns of the Yinggelike nephrite [60]. 
The nephrite samples contain $\delta^{18} \mathrm{O}=5.3$ to $7.4 \%$ ond $\delta \mathrm{D}=-74.9$ to $-86.7 \%$, exhibiting a narrow range. Hydrogen and oxygen isotope compositions in the nephrite from Yingelike and other well-known nephrite deposits worldwide are summarized in Table 6 and illustrated in Figure 9.

Table 6. Hydrogen and oxygen isotope compositions of the nephrite from Yingelike and other deposits.

\begin{tabular}{|c|c|c|c|c|c|}
\hline Samples & $\delta \mathrm{D}$ & $\delta^{18} \mathrm{O}$ & & & \\
\hline $\mathrm{H}-3$ & -74.9 & 5.3 & & & \\
\hline $\mathrm{H}-4$ & -78.0 & 7.4 & & & \\
\hline $\mathrm{H}-5$ & -86.7 & 5.4 & & & \\
\hline Average & -79.9 & 6.0 & & & \\
\hline Types & \multicolumn{2}{|c|}{ Dolomite-related } & \multicolumn{3}{|c|}{ Serpentinite-related } \\
\hline Deposits & $\delta \mathrm{D}$ & $\delta^{18} \mathrm{O}$ & Deposits & $\delta \mathrm{D}$ & $\delta^{18} \mathrm{O}$ \\
\hline Kunlun, China $^{1}$ & $-108 \sim-124$ & $0.5 \sim 2.3$ & Chara Jelgra River, Siberia ${ }^{1}$ & -39 & 6.9 \\
\hline Cowell, Australia ${ }^{1}$ & -57 & 3.4 & Red Mountain, New Zealand ${ }^{1}$ & -54 & 7.5 \\
\hline Wyoming, USA ${ }^{1}$ & -56 & 1.5 & Mt. Ogden, Canada ${ }^{1}$ & -52 & 9.6 \\
\hline Chuncheon, Korea $^{1}$ & $-118 \sim-105$ & $-9.9 \sim-7.9$ & Shulaps Range, Canada ${ }^{1}$ & -49 & 8.4 \\
\hline Alamas, Xinjiang, China ${ }^{2}$ & $-95 \sim-75$ & $3.2 \sim 6.2$ & Fengtien, China ${ }^{1}$ & $-68 \sim-33$ & $4.5 \sim 5.3$ \\
\hline Hetian placer nephrite, China $^{3}$ & $-72.4 \sim-55.7$ & $1.1 \sim 5.6$ & Qinghai, China $^{7}$ & $-60 \sim-56$ & $8.1 \sim 8.6$ \\
\hline Złoty Stok, SW Poland 4 & $-77.2 \sim-74.6$ & $8.3 \sim 10.4$ & Pakistan ${ }^{7}$ & $-56 \sim-43$ & $13.2 \sim 13.4$ \\
\hline Tashisayi, Xinjiang, China ${ }^{5}$ & $-89.3 \sim-82.5$ & $3.0 \sim 6.5$ & Jordanów, SW Poland ${ }^{8}$ & -61 & 6.1 \\
\hline Apocarbonate nephrite, Russia ${ }^{6}$ & $-179 \sim-119$ & $-17 \sim-15$ & & & \\
\hline
\end{tabular}

Data source: ${ }^{1}$ From [31]; ${ }^{2}$ From $[18] ;{ }^{3}$ from $[19] ;{ }^{4}$ from [36]; ${ }^{5}$ from [38]; ${ }^{6,7}$ from [61]; ${ }^{8}$ from [32].

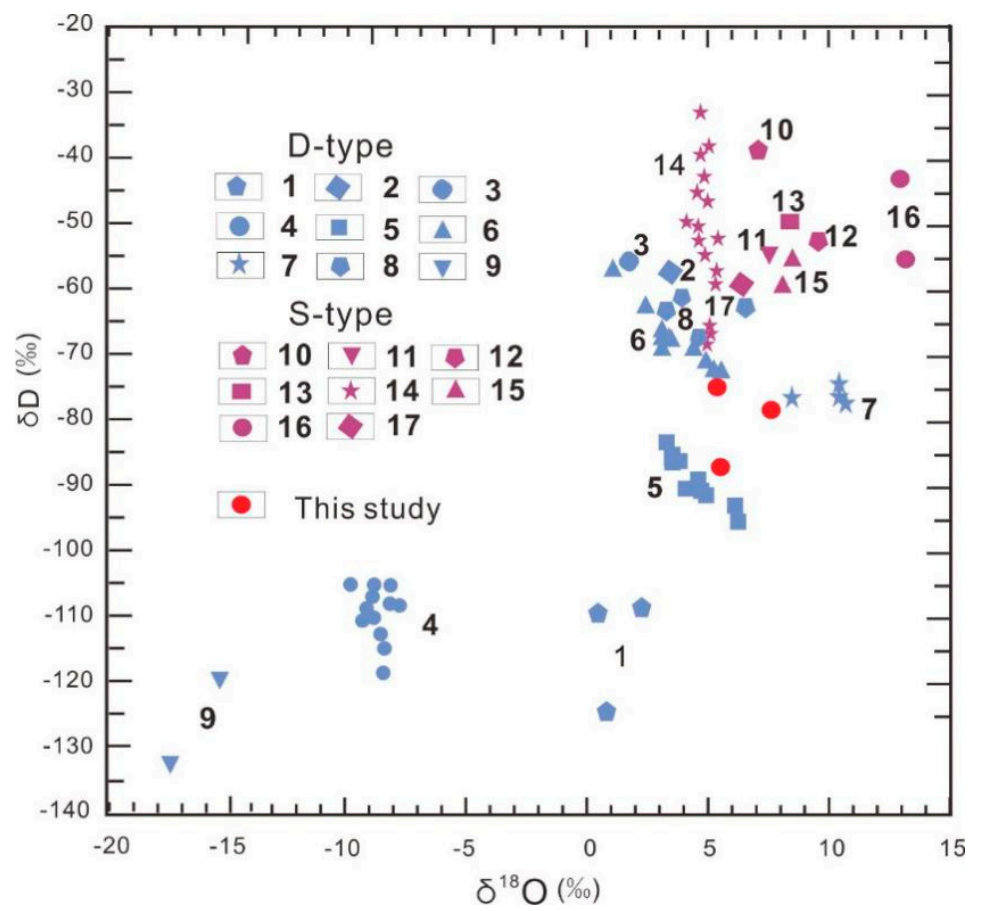

Figure 9. $\delta \mathrm{D}$ and $\delta^{18} \mathrm{O}$ of nephrites from Yinggelike (this study) and other dolomite-related (D-type) deposits: (1) Kunlun $(n=3)$; (2) Cowell $(n=1)$; (3) Wyoming $(n=1)$; (4) Chuncheon $(n=12)$; (5) Alamas $(n=12)$; (6) Hetian placer nephrite $(n=10)$; (7) Złoty Stok $(n=4)$, (8) Tashisayi $(n=4)$;

(9) Apocarbonate nephrite $(n=2)$, and serpentinite-related (S-type) ones: (10) Chara Jelgra River $(n=1)$;

(11) Red Mountain $(n=1)$; (12) Mount Ogden ( $n=1)$; (13) Shulaps Range $(n=1)$; (14) Fengtien $(n=16)$,

(15) Qinghai $(n=2)$; (16) Pakistan $(n=2)$; (17) Jordanów, SW Poland $(n=1)$ (see Table 6 for details).

\section{Discussion}

\subsection{Dolomite-Related Origin}

Field investigation shows that the Yinggelike nephrite orebody occurs in the dolomitic marble near the intruding granitoids (Figure 2), which suggests that the Yinggelike nephrite deposit is of a 
dolomite-related type. This conclusion is supported by the mineralogy, bulk-rock chemistry, and $\mathrm{O}$ and $\mathrm{H}$ isotopes characteristics of the nephrite.

From the mineralogical characteristics of nephrites of different genetic origins around the world (summarized in Table 7), major differences are present between the dolomite-related (D-type) and serpentinite-related (S-type) nephrites: the main mineral of D-type nephrites is tremolite ( $>95 \mathrm{vol.} \%$ ), although diopside exists in almost all D-type nephrites and is considered to be related to the nephrite formation $[18,19,22]$. Minor minerals mainly include actinolite, carbonate, epidote, clinozolisite, apatite, allanite, quartz, etc. Almost all the minerals in the D-type nephrites were iron-poor [18,19,22,37,62-65]. In contrast, S-type nephrites mainly contained tremolite-actinolite series minerals, with minor grossular, spinel, chlorite, serpentine, chromite, magnetite, magnesite, monazite, maucherite, etc., but no carbonate minerals [22,33,66-71]. Iron-rich minerals, such as magnetite and chromite, are highly common. S-type nephrite has commonly black impurities (chromite, spinel or other opaque minerals) visible to the naked eye. Our petrographic observations, whole-rock geochemical and mineral EMPA data all suggest that the Yinggelike nephrite is composed predominantly of tremolite ( $>98 \mathrm{vol} . \%)$, with minor calcite but no serpentine or Fe-rich minerals such as magnetite and chromite. The occurrence of tremolite in carbonate pseudomorphs points to metasomatic replacement processes (Figures $5 b$ and $6 a$ ), similar to that proposed for the typical D-type Chuncheon nephrite deposit [64].

Table 7. Mineralogical characteristics of the world's major D-/S-type nephrite deposits.

\begin{tabular}{|c|c|c|c|}
\hline Types & Deposits & Major Minerals & Minor Minerals \\
\hline \multirow{7}{*}{ D-type } & Złoty Stok, SW Poland ${ }^{1}$ & $\begin{array}{c}\text { Type 1: actinolite, diopside } \\
\text { Type 2: tremolite-actinolite (>90\%) }\end{array}$ & $\begin{array}{l}\text { Type 1: lollingite, carbonates, quartType 2: } \\
\text { diopside, calcite, quartz, As-minerals, } \\
\text { apatite, scheelite, allanite }\end{array}$ \\
\hline & Alamas, Xinjiang, China ${ }^{2}$ & Tremolite (>98\%) & Diopside, actinolite, calcite \\
\hline & Hetian placer nephrite, $\mathrm{China}^{3}$ & Tremolite $(>95 \%)$ & Diopside, calcite, allanite, zircon, rutile \\
\hline & Chuncheon, Korea ${ }^{4}$ & Tremolite ( 98\%) & $\begin{array}{l}\text { Diopside, serpentine, clinochlore, } \\
\text { carbonates, apatite }\end{array}$ \\
\hline & Qinghai, China ${ }^{5}$ & Tremolite (>95\%) & $\begin{array}{l}\text { Diopside, calcite, epidote, clinozolisite, } \\
\text { titanite, actinolite }\end{array}$ \\
\hline & Tiantai, Xinjiang, China ${ }^{6}$ & Tremolite (>95\%) & $\begin{array}{l}\text { Diopside, dolomite, titanite, epidote, } \\
\text { magnetite, apatite, limonite }\end{array}$ \\
\hline & Liyang, Jiangsu, China ${ }^{7}$ & Tremolite ( 99\%) & Diopside, apatite, carbonates \\
\hline \multirow{7}{*}{ S-type } & Jordanów, SW Poland ${ }^{8}$ & $\begin{array}{l}\text { Tremolite (97.2-89.8\%), diopside } \\
(4.0-5.7 \%) \text { and chlorite }(3.8-8.1 \%)\end{array}$ & $\begin{array}{c}\text { Grossular, prehnite, opaque spinel, } \\
\text { antigorite, hydrogrossular, titanite, zircon, } \\
\text { apatite, monazite }\end{array}$ \\
\hline & $\begin{array}{l}\text { Mount Ogden area, Central } \\
\text { British Columbia }^{9}\end{array}$ & $\begin{array}{l}\text { Actinolite-tremolite }(>90 \%) \text {, serpentine } \\
\qquad(<10 \%) \text {, talc }(\sim 6 \%)\end{array}$ & $\begin{array}{c}\text { Spinel, titanite, ilmenite, } \\
\text { hematite, chlorite }\end{array}$ \\
\hline & Qinghai, China ${ }^{10}$ & Tremolite, minor actinolite & $\begin{array}{c}\text { Diopside, chlorite, zoisite, rutile, zircon, } \\
\text { serpentine, magnetite, magnesite, } \\
\text { chromite, augite }\end{array}$ \\
\hline & Manasi, Xinjiang, China ${ }^{11}$ & Tremolite-actinolite (75-90\%) & Chromite, kirschsteinite, chlorite \\
\hline & South Island, New Zealand ${ }^{12}$ & Mainly tremolite, less actinolite & Albite, Chlorite, diopside, Maucherite \\
\hline & Hualian, Taiwan, China ${ }^{13}$ & Tremolite-actinolite & Grossular, chromite, chlorite \\
\hline & East Sayan, Siberia, Russia ${ }^{14}$ & Tremolite & Chlorite, chromite \\
\hline
\end{tabular}

The $\mathrm{Fe} /(\mathrm{Fe}+\mathrm{Mg})$ ratios can distinguish between D- and S-type nephrites, with those of D-type nephrites often falling below 0.06 (considerably lower than S-type) [2,72,73]. The Yinggelike nephrite has $\mathrm{Fe} /(\mathrm{Fe}+\mathrm{Mg}$ ) ratios of 0.02 to 0.05 , thus falling within the D-type field. In addition, the Yinggelike nephrites have very low concentrations of $\mathrm{Cr}(0.81-34.68 \mathrm{ppm}), \mathrm{Co}(1.10-2.91 \mathrm{ppm})$, and $\mathrm{Ni}$ $(0.52-20.15 \mathrm{ppm})$, which are consistent with a D-type origin $(\mathrm{Cr}=1.9-179.0 \mathrm{ppm}, \mathrm{Co}=0.5-9.8 \mathrm{ppm}$, $\mathrm{Ni}=0.05-470.7 \mathrm{ppm})$, but inconsistent with an S-type one $(\mathrm{Cr}=719-2812 \mathrm{ppm}, \mathrm{Co}=42-260 \mathrm{ppm}$, $\mathrm{Ni}=959-1898 \mathrm{ppm})$ [32]. The low compatible trace element $(\mathrm{Cr}, \mathrm{Co}, \mathrm{Ni})$ contents for the Yinggelike nephrite are likely inherited from its protoliths, i.e., dolomitic marble instead of serpentinized ultramafic rocks.

Previous study has summarized the REE characteristics of nephrite from different deposits [74]. REE features of nephrites are likely co-influenced by their geological environment and petrogenetic processes, and can thus be used to distinguish nephrites of different genetic origins [74]. The Yinggelike 
nephrite shares similar REE patterns to many D-type nephrites (e.g., Alamas and Qinghai ones; [18,22]), including moderate LREE enrichment $\left(\mathrm{La}_{\mathrm{N}} / \mathrm{Sm}_{\mathrm{N}}=1.72-4.04\right)$, nearly flat HREE patterns $\left(\mathrm{Gd}_{\mathrm{N}} / \mathrm{Yb}_{\mathrm{N}}=\right.$ 0.13-2.62), strong to moderate negative Eu anomalies $\left(\mathrm{Eu} / \mathrm{Eu}^{*}=0.04-0.67\right)$, and low $\Sigma \mathrm{REE}$ contents (2.16-11.25 ppm) (Figure 8; Table A2). Meanwhile, these REE characteristics of the Yinggelike nephrite are different from many S-type nephrites, which are featured by left-inclining REE patterns, strong positive Eu anomalies, significantly elevated $\Sigma$ REE (2200-3000 ppm) and LREE/HREE (151-326) that reflect a serpentinite-origin (e.g., Qinghai, Hualian, and Manasi nephrites) [74].

As summarized by the compilation of data presented in Table 6 and Figure 9. D-type nephrites tend to have relatively low $\delta^{18} \mathrm{O}$ and $\delta \mathrm{D}$ values, and vice versa for S-type ones albeit some overlapping. The $\delta^{18} \mathrm{O}(5.3-7.4 \%$ o) and $\delta \mathrm{D}(-74.9$ to $-86.7 \%$ o) values of the Yinggelike nephrite fall closer to many D-type nephrite deposits (esp. Alamas, Hetian and Złoty Stok) than the S-type ones in the $\delta^{18} \mathrm{O} v \mathrm{vs} . \delta \mathrm{D}$ diagram. The isotopic difference from some other D-type nephrite deposits (e.g., Chuncheon; Russia) may have been caused by different formation temperatures, fluid/rock ratios, and/or protolith isotope inheritance $[25,31]$.

\subsection{Metasomatic Process in Nephrite}

Based on mineral paragenetic relationships obtained from this and previous studies $[1,16,18,19,75]$, three mineral crystallization stages are recognized at Yinggelike.

Stage I is associated with granitoid intrusion (Figure 1c). Representative mineral formed during this stage is diopside (Figure 5e). Anhydrous andesine, titanite, and zircon were probably formed at this stage (Figure $6 \mathrm{f}, \mathrm{h}, \mathrm{i})$. The prograde metasomatic process that formed the diopside can be expressed as follows:

$$
\mathrm{CaMg}\left(\mathrm{CO}_{3}\right)_{2} \text { (dolomite) }+2 \mathrm{SiO}_{2} \text { (aq) } \rightarrow \mathrm{CaMgSi}_{2} \mathrm{O}_{6} \text { (diopside) }+2 \mathrm{CO}_{2} \text { (aq.) }
$$

Stage II features the formation of fine and randomly oriented tremolite fibers, which contributes to the gem-quality. This stage is related to the retrograde hydrothermal alteration, during which various mineral replacement reactions had occurred. The main formation processes are as follows:

(1) The dolomitic marble was replaced by tremolite. This is a common explanation for the formation of nephrite [1].

$$
\begin{gathered}
5 \mathrm{CaMg}\left(\mathrm{CO}_{3}\right)_{2} \text { (dolomite) }+8 \mathrm{SiO}_{2} \text { (aq.) }+\mathrm{H}_{2} \mathrm{O} \rightarrow \mathrm{Ca}_{2} \mathrm{Mg}_{5} \mathrm{Si}_{8} \mathrm{O}_{22}(\mathrm{OH})_{2} \text { (tremolite) }+ \\
3 \mathrm{CaCO}_{3} \text { (calcite) }+7 \mathrm{CO}_{2} \text { (aq.) }
\end{gathered}
$$

Both tremolite Tr-I and Tr-II can be formed in the reaction presented in Equation (2). The coarsegrained Tr-I formed were likely replaced by or recrystallized into later Tr-II (Figure 5b). The lower formation temperature (plus possible rapid cooling led by meteoric fluid incursion) likely deprived Tr-II of sufficient time to crystallize, which generated its characteristic micro-/cryptocrystalline texture.

(2) Stage I diopside was partially replaced by tremolite along its grain boundary and cracks (Figure 5e), either directly by Tr-II or by Tr-I and then Tr-II (Figure $6 \mathrm{~b}$ ). This process can be expressed in the following equation:

$$
5 \mathrm{CaMgSi}_{2} \mathrm{O}_{6} \text { (diopside) }+\mathrm{H}_{2} \mathrm{O} \rightarrow \mathrm{Ca}_{2} \mathrm{Mg}_{5}\left(\mathrm{Si}_{4} \mathrm{O}_{11}\right)_{2}(\mathrm{OH})_{2}(\text { tremolite })+3 \mathrm{Ca}^{2+}+6 \mathrm{SiO}_{2}
$$

Unlike most other nephrite deposits in the Hetian nephrite belt [17-21], pargasite is common in the Yinggelike nephrite. In some cases, Stage I diopside was replaced by pargasite, which was in turn replaced by $\mathrm{Tr}$-II (Figure 6c). The $\mathrm{Al}$ and Na contents in pargasite are higher than those in diopside, which indicates that the process of diopside replacement by paragasite may have been the result of Aland Na-rich hydrothermal alteration (Table 3).

(3) Isolated calcite grains in nephrites are suggested to be the products of Reaction (2) $[1,18,19,22]$. At Yinggelike, isolated calcite grains were not observed, and calcite occurs mainly as late-stage veins. 
We suggest that the Stage II isolated calcite grains may have been completely taken up by the formation of fine-grained Tr-II, as expressed in the following equation:

$$
\begin{gathered}
2 \mathrm{CaCO}_{3}(\text { calcite })+5 \mathrm{Mg}^{2+}+8 \mathrm{SiO}_{2}+6 \mathrm{H}_{2} \mathrm{O} \rightarrow \mathrm{Ca}_{2} \mathrm{Mg}_{5}\left(\mathrm{Si}_{4} \mathrm{O}_{11}\right)_{2}(\mathrm{OH})_{2}(\text { tremolite })+ \\
10 \mathrm{H}^{+}+2 \mathrm{CO}_{2}
\end{gathered}
$$

In the above equation, the tremolite formation requires $\mathrm{Mg}$, which is likely derived from the dolomitic marble wallrocks leached by hydrothermal fluids, as first proposed by [75]. With the gradual consumption of calcite and production of $\mathrm{H}^{+}$ions, the hydrothermal fluid may have become more acidic. This likely led to further carbonate decomposition and produced an excess of $\mathrm{Ca}^{2+}$ ions, which may have formed other Ca-rich intermediate reaction products such as epidote, allanite, and prehnite (Figures $5 \mathrm{f}-\mathrm{g}$ and $6 \mathrm{e}-\mathrm{g}$ ).

Stage III, characterized by calcite veins (Figure 5h), is related to the hydrothermal processes after nephrite formation.

\subsection{Gemological Significance}

The general preference of yellow color (associated with royalty) in many East Asian cultures has placed yellowish nephrite (and to a lesser extent white one, which is known "suet nephrite" in China) above nephrites of other colors [16]. Nevertheless, yellow nephrite is very rare, and is only reported in South Korea and the Qinghai and Liaoning provinces in China $[22,64,76]$. This makes yellow nephrite even more precious.

The factors that control the coloring of nephrites are still not fully understood, and thus we compare different yellowish-colored Yinggelike nephrite samples in this study. The colors of our samples can be broadly divided into a more greenish-yellow (Q-1, Q-2, Q-3) to a light-yellow (Q-4, Q-5, Q-6) group (Figure 4). EMPA and bulk-rock XRF/ICP-MS analyses show that all the samples were mainly composed of tremolite, and were almost free of impurities (Tables 2 and 4). Therefore, the main chromogenic mineral was tremolite. The high contents of chromogenic cations such as $\mathrm{Fe}^{2+}, \mathrm{Fe}^{3+}$, Mn and Ti suggest that they may be important in determining the Yinggelike nephrite color. Some workers have suggested that the green color of nephrite is mainly caused by the substitution of $\mathrm{Fe}^{2+}$ for $\mathrm{Mg}^{2+}$, and the yellow color may be caused primarily by $\mathrm{Fe}^{3+}$ and $\mathrm{Mn}[15,18,19,77-80]$. Titanium by itself is not known as a coloration factor for most terrestrial minerals, and as for nephrite, it affects the coloration of the blue-violet variety [79]. We therefore propose that $\mathrm{Fe}^{2+}, \mathrm{Fe}^{3+}$, and $\mathrm{Mn}$ are the main chromogenic factors for the nephrite. The greenish color of nephrite is related to $\mathrm{Fe}^{2+}$, while the yellowish color is related to $\mathrm{Fe}^{3+}$ and $\mathrm{Mn}$. In addition, the greenish-yellow group has markedly higher $\mathrm{Fe}^{3+}$, and moderately higher $\mathrm{Fe}^{2+}$ and Mn contents than those of the light-yellow group, which indicates that $\mathrm{Fe}^{3+}$ is more important in giving the yellow color than $\mathrm{Mn}$.

Generally, the finer the tremolite grains, the better the jade quality. Our petrographic observations indicate that the Yinggelike nephrite consists mainly of fine-grained tremolite (especially Tr-II), the aggregates of which give the nephrite a fine and compact texture (Figure 4). The randomly oriented bundles of twisted micro-fibers in the nephrite also result in its extreme toughness (Figure 5c). This toughness, combined with a relatively high Mohs hardness (5.8-6.1) (Table 1), makes the Yingglike nephrite a precious carving material.

\section{Conclusions}

In this paper, we presented the first systematic study of field geological occurrence, mineralogy, bulk-rock chemistry, and stable $(\mathrm{O}$ and $\mathrm{H})$ isotope characteristics of nephrite from Yinggelike deposit in the Altyn Tagh Mountains (NW China). These data contribute to the overall understanding of the Yinggelike nephrite deposit.

The nephrite is dominated by tremolite, with minor minerals such as pargasite, diopside, epidote, allanite, prehnite, andesine, titanite, zircon, and calcite. Geochemical analyses shows that 
the nephrite samples have low bulk-rock $\mathrm{Fe} /(\mathrm{Fe}+\mathrm{Mg}$ ) values and $\mathrm{Cr}, \mathrm{Co}$, and $\mathrm{Ni}$ concentrations. The chondrite-normalized REE patterns of most samples exhibit strong to moderate negative Eu anomalies with moderate LREE enrichment, nearly flat HREE, and low $\Sigma$ REE contents. The mineral and geochemical characteristics of Yinggelike nephrite are consistent with those of the dolomite-related ones.

Mineral paragenetic relationships define three mineral crystallization stages for the Yinggelike nephrite: (1) diopside formed by prograde metasomatism; (2) nephrite jade formed by retrograde metasomatism and replacement of Stage I anhydrous minerals; (3) hydrothermal alteration after the nephrite formation. Features of transition metal contents indicate that the color of the Yinggelike nephrite is probably controlled by $\mathrm{Fe}^{2+}, \mathrm{Fe}^{3+}$, and Mn. Our new mineralogical and geochemical results on the Yinggelike nephrite provide better constraints on the formation of other nephrite deposits in the Altyn Tagh Mountains, and can facilitate future nephrite prospecting and research in the region.

Author Contributions: Conceptualization, Y.J. and G.S.; methodology, Y.J. and L.X.; validation, Y.J., G.S. and L.X.; formal analysis, Y.J.; investigation, Y.J., G.S. and L.X; resources, G.S. and X.L.; writing - original draft preparation, Y.J.; writing-review and editing, Y.J. and G.S; supervision, G.S.; project administration, G.S.; funding acquisition, G.S. All authors have read and agreed to the published version of the manuscript.

Funding: This study was funded by the National Science Foundation of China (41688103), the Strategic Priority Research Program of the Chinese Academy of Sciences (Grant No. XDA20070304).

Acknowledgments: We appreciate Shoucheng Wang, Shiqi Wang (PKU), and Xiaojin Yu (USTB) for their kind field supports. We also thank Ji Zhang, $\mathrm{Xu} \mathrm{Li}$, and Kong Gao for the fruitful discussion. Constructive reviews by John Valley has greatly enhanced the manuscript. The Editors and two anonymous reviewers are thanked for their insightful comments, which significantly enhanced the manuscript.

Conflicts of Interest: The authors declare no conflict of interest.

\section{Appendix A}

Table A1. Chemical compositions of epidote, allanite, andesine, prehnite, titanite, and calcite in the Yinggelike nephrite, Xinjiang, NW China (wt.\%).

\begin{tabular}{|c|c|c|c|c|c|c|c|c|c|c|}
\hline Samples & H-5-1 & H-5-2 & H-3 & H-3 & H-3 & H-3-1 & H-3-2 & H-1 & H-3 & H-5 \\
\hline Minerals & Allanite & Allanite & Epidote & Epidote & Andesine & Prehnite & Prehnite & Titanite & Calcite & Zircon \\
\hline $\mathrm{SiO}_{2}$ & 35.64 & 36.25 & 37.75 & 37.14 & 61.19 & 44.85 & 44.75 & 30.59 & 0.01 & 31.52 \\
\hline $\mathrm{ZrO}_{2}$ & n.d. & n.d. & n.d. & n.d. & n.d. & n.d. & n.d. & n.d. & n.d. & 65.54 \\
\hline $\mathrm{UO}_{3}$ & n.d. & n.d. & n.d. & n.d. & n.d. & n.d. & n.d. & n.d. & n.d. & 1.79 \\
\hline $\mathrm{TiO}_{2}$ & 0.00 & 0.19 & 0.08 & 0.02 & 0.00 & 0.05 & 0.02 & 36.85 & 0.00 & n.d. \\
\hline $\mathrm{Al}_{2} \mathrm{O}_{3}$ & 23.22 & 24.73 & 28.05 & 28.30 & 24.32 & 22.86 & 23.53 & 2.13 & 0.00 & n.d. \\
\hline $\mathrm{FeO}$ & 7.24 & 5.11 & 3.78 & 3.40 & 0.13 & 0.17 & 0.22 & 0.04 & 0.03 & n.d. \\
\hline $\mathrm{Cr}_{2} \mathrm{O}_{3}$ & 0.00 & 0.00 & 0.02 & 0.04 & 0.05 & 0.00 & 0.00 & 0.05 & 0.01 & n.d. \\
\hline $\mathrm{MnO}$ & 0.32 & 0.15 & 0.05 & 0.00 & 0.00 & 0.00 & 0.01 & 0.02 & 0.06 & n.d. \\
\hline $\mathrm{MgO}$ & 0.73 & 2.11 & 0.52 & 0.44 & 0.04 & 0.14 & 0.07 & 0.02 & 0.25 & n.d. \\
\hline $\mathrm{CaO}$ & 20.11 & 18.14 & 25.72 & 25.71 & 7.27 & 27.23 & 27.01 & 29.28 & 55.88 & n.d. \\
\hline $\mathrm{Na}_{2} \mathrm{O}$ & 0.01 & 0.00 & 0.04 & 0.00 & 7.23 & 0.01 & 0.05 & 0.01 & 0.06 & 1.18 \\
\hline $\mathrm{K}_{2} \mathrm{O}$ & 0.02 & 0.02 & 0.00 & 0.02 & 0.12 & 0.02 & 0.01 & 0.03 & 0.00 & n.d. \\
\hline $\mathrm{NiO}$ & 0.00 & 0.00 & 0.00 & 0.05 & 0.01 & 0.02 & 0.03 & 0.01 & 0.03 & n.d. \\
\hline $\mathrm{La}_{2} \mathrm{O}_{3}$ & 2.58 & 2.65 & n.d. & n.d. & n.d. & n.d. & n.d. & n.d. & n.d. & n.d. \\
\hline $\mathrm{Ce}_{2} \mathrm{O}_{3}$ & 5.85 & 5.96 & n.d. & n.d. & n.d. & n.d. & n.d. & n.d. & n.d. & n.d. \\
\hline $\mathrm{Pr}_{2} \mathrm{O}_{3}$ & 0.43 & 1.20 & n.d. & n.d. & n.d. & n.d. & n.d. & n.d. & n.d. & n.d. \\
\hline $\mathrm{Nd}_{2} \mathrm{O}_{3}$ & 2.03 & 2.37 & n.d. & n.d. & n.d. & n.d. & n.d. & n.d. & n.d. & n.d. \\
\hline Total & 98.19 & 98.88 & 95.99 & 95.12 & 100.36 & 95.35 & 95.70 & 99.02 & 56.34 & 100.03 \\
\hline
\end{tabular}

0.00—concentration below the detection limit; n.d.—not detected. 
Table A2. Trace element compositions of the nephrite samples collected from the Yinggelike open pit, Xinjiang, NW China (ppm).

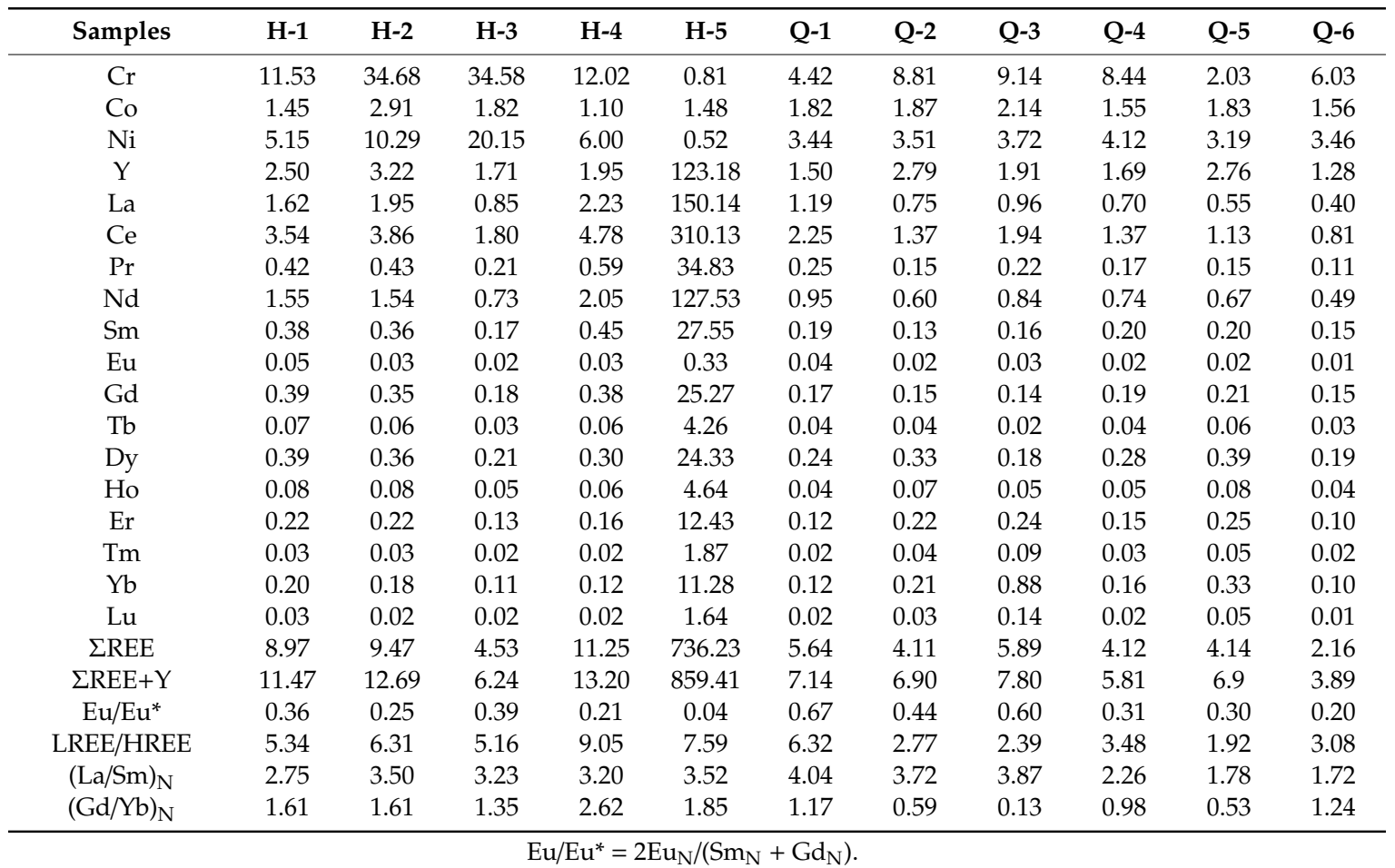

\section{References}

1. Harlow, G.E.; Sorensen, S.S. Jade (nephrite and jadeitite) and serpentinite: Metasomatic connections. Int. Geol. Rev. 2005, 47, 113-146. [CrossRef]

2. Wen, G.; Jing, Z.C. Chinese Neolithic Jade: A Preliminary Geoarchaeological Study. Geoarchaeology 1992, 7, 251-275. [CrossRef]

3. D'Amico, C.; Starnini, E.; Gasparotto, G.; Ghedini, M. Eclogites, jades and other HP-metaophiolites employed for prehistoric polished stone implements in Italy and Europe. Period. Mineral. 2004, 73, 17-42.

4. Middleton, A. Jade-geology and mineralogy. In Gems; O’Donoghue, M., Ed.; Elsevier Ltd.: Oxford, UK, 2006; pp. 332-354.

5. Losey, R.J.; Bazaliiskii, V.I.; Garvie-Lok, S.; Germonpré, M.; Leonard, J.A.; Allen, A.L.; Anne Katzenberg, M.; Sablin, M.V. Canids as persons: Early Neolithic dog and wolf burials, Cis-Baikal, Siberia. J. Anthropol. Archaeol. 2011, 30, 174-189. [CrossRef]

6. Tsydenova, N.; Morozov, M.V.; Rampilova, M.V.; Vasil'ev, Y.A.; Matveeva, O.P.; Konovalov, P.B. Chemical and spectroscopic study of nephrite artifacts from Transbaikalia, Russia: Geological sources and possible transportation routes. Quat. Int. 2015, 355, 114-125. [CrossRef]

7. Kostov, R.I. Gemmological significance of the prehistoric Balkan "nephrite culture" (cases from Bulgaria). Annu. Univ. Min. Geol. 2005, 48, 91-94.

8. Kostov, R.I.; Protochristov, C.; Stoyanov, C.; Csedreki, L.; Simon, A.; Szikszai, Z.; Uzonyi, I.; Gaydarska, B.; Chapman, J. Micro-PIXE geochemical fingerprinting of nephrite neolithic artifacts from Southwest Bulgaria. Geoarchaeology 2012, 27, 457-469. [CrossRef]

9. Péterdi, B.; Szakmány, G.; Judik, K.; Dobosi, G.; Kasztovszky, Z.; Szilágyi, V.; Maróti, B.; Bendõ, Z.; Gil, G. Petrographic and geochemical investigation of a stone adze made of nephrite from the balatonoszöd-Temetoi dulo site (Hungary), with a review of the nephrite occurrences in Europe (especially in Switzerland and in the Bohemian Massif). Geol. Q. 2014, 58, 181-192. [CrossRef]

10. Chen, T.-H.; Calligaro, T.; Pagès-Camagna, S.; Menu, M. Investigation of Chinese archaic jade by PIXE and $\mu$ Raman spectrometry. Appl. Phys. A 2004, 79, 177-180. [CrossRef] 
11. Sax, M.; Meeks, N.D.; Michaelson, C.; Middleton, A.P. The identification of carving techniques on Chinese Jade. J. Archaeol. Sci. 2004, 31, 1413-1428. [CrossRef]

12. Derevianko, A.P.; Olsen, J.W.; Tseveendorj, D.; Gladyshev, S.A.; Nokhrina, T.I.; Tabarev, A.V. New insights into the archaeological record at Chikhen Agui Rockshelter (Mongolia). Archaeol. Ethnol. Anthropol. Eurasia 2008, 34, 2-12. [CrossRef]

13. Fournelle, J.; Law, R.; Konishi, H. A nephrite jade amulet from Harappa: Implications for long-distance contacts in the Harappan Period. In Proceedings of the 39th Annual Conference on South Asia, New York, NY, USA, 14-17 October 2010.

14. Hung, H.-C.; Iizuka, Y.; Bellwood, P.; Nguyen, K.D.; Bellina, B.; Silapanth, P.; Dizon, E.; Santiago, R.; Datan, I.; Manton, J.H. Ancient jades map 3,000 years of prehistoric exchange in Southeast Asia. Proc. Natl. Acad. Sci. USA 2007, 104, 19745-19750. [CrossRef] [PubMed]

15. Wilkins, C.J.; Tennant, W.C.; Williamson, B.E.; McCammon, C.A. Spectroscopic and related evidence on the coloring and constitution of New Zealand jade. Am. Mineral. 2003, 88, 1336-1344. [CrossRef]

16. Tang, Y.L.; Chen, B.Z.; Jiang, R.H. Chinese Hetian Nephrite; Xinjiang People's Publishing House: Xinjiang, China, 1994; pp. 103-206. (In Chinese with English abstract)

17. Liu, Y.; Deng, J.; Shi, G.H.; Lu, T.J.; He, H.Y.; Ng, Y.-N.; Shen, C.H.; Yang, L.Q.; Wang, Q.F. Chemical Zone of Nephrite in Alamas, Xinjiang, China. Resour. Geol. 2010, 60, 249-259. [CrossRef]

18. Liu, Y.; Deng, J.; Shi, G.H.; Yui, T.-F.; Zhang, G.B.; Abuduwayiti, M.; Yang, L.Q.; Sun, X. Geochemistry and petrology of nephrite from Alamas, Xinjiang, NW China. J. Asian Earth Sci. 2011, 42, 440-451. [CrossRef]

19. Liu, Y.; Deng, J.; Shi, G.H.; Sun, X.; Yang, L.Q. Geochemistry and petrogenesis of placer nephrite from Hetian, Xinjiang, Northwest China. Ore Geol. Rev. 2011, 41, 122-132. [CrossRef]

20. Liu, Y.; Zhang, R.Q.; Abuduwayiti, M.; Wang, C.; Zhang, S.P.; Shen, C.H.; Zhang, Z.Y.; He, M.Y.; Zhang, Y.; Yang, X.D. SHRIMP U-Pb zircon ages, mineral compositions and geochemistry of placer nephrite in the Yurungkash and Karakash River deposits, West Kunlun, Xinjiang, Northwest China: Implication for a magnesium skarn. Ore Geol. Rev. 2016, 72, 699-727. [CrossRef]

21. Liu, Y.; Zhang, R.Q.; Zhang, Z.Y.; Shi, G.H.; Zhang, Q.C.; Abuduwayiti, M.; Liu, J.H. Mineral inclusions and SHRIMP U-Pb dating of zircons from the Alamas nephrite and granodiorite: Implications for the genesis of a magnesian skarn deposit. Lithos 2015, 212-215, 128-144. [CrossRef]

22. Yu, H.Y.; Wang, R.C.; Guo, J.C.; Li, J.G.; Yang, X.W. Study of the minerogenetic mechanism and origin of Qinghai nephrite from Golmud, Qinghai, Northwest China. Sci. China Earth Sci. 2016, 59, 1597-1609. [CrossRef]

23. Yin, Z.W.; Jiang, C.; Santosh, M.; Chen, Y.M.; Bao, Y.; Chen, Q.L. Nephrite Jade from Guangxi Province, China. Gems Gemol. 2014, 50, 228-235. [CrossRef]

24. Zhang, C.; Yu, X.; Jiang, T. Mineral association and graphite inclusions in nephrite jade from Liaoning, northeast China: Implications for metamorphic conditions and ore genesis. Geosci. Front. 2019, 10, 425-437. [CrossRef]

25. Burtseva, M.V.; Ripp, G.S.; Posokhov, V.F.; Zyablitsev, Y.A.; Murzintseva, A.E. The sources of fluids for the formation of nephritic rocks of the southern folded belt of the Siberian. Craton. Dokl. Earth Sci. 2015, 460, 82-86. [CrossRef]

26. Burtseva, M.V.; Ripp, G.S.; Posokhov, V.F.; Murzintseva, A.E. Nephrites of East Siberia: Geochemical features and problems of genesis. Russ. Geol. Geophys. 2015, 56, 402-410. [CrossRef]

27. Leaming, S.F. Jade in Canada; Geological Survey of Canada: Ottawa, ON, Canada, 1978; pp. 1-59.

28. Adams, C.J.; Beck, R.J.; Campbell, H.J. Characterisation and origin of New Zealand nephrite jade using its strontium isotopic signature. Lithos 2007, 97, 307-322. [CrossRef]

29. Cox, S.C.; Nibourel, L. Bedload composition, transport and modification in rivers of Westland, New Zealand, with implications for the distribution of alluvial pounamu (jade). N. Z. J. Geol. Geophys. 2015, 58, 154-175. [CrossRef]

30. Cooper, A.F. Nephrite and Metagabbro in the Haast Schist at Muddy Creek, Northwest Otago, New Zealand. N. Z. J. Geol. Geophys. 1995, 38, 325-332. [CrossRef]

31. Yui, T.-F.; Kwon, S.-T. Origin of a Dolomite-Related Jade Deposit at Chuncheon, Korea. Econ. Geol. 2002, 97, 593-601. [CrossRef] 
32. Gil, G.; Barnes, J.D.; Boschi, C.; Gunia, P.; Szakmány, G.; Bendő, Z.; Raczynski, P.; Petérdi, B. Origin of serpentinite-related nephrite from Jordanów and adjacent areas (SW Poland) and its comparison with selected nephrite occurrences. Geol. Q. 2015, 59, 457-472. [CrossRef]

33. Gil, G. Petrographic and microprobe study of nephrites from Lower Silesia (SW Poland). Geol. Q. 2013, 57, 395-404. [CrossRef]

34. Yui, T.-F.; Yeh, H.-W.; Lee, C.W. Stable isotope studies of nephrite deposits from Fengtien, Taiwan. Geochim. Cosmochim. Acta 1988, 52, 593-602. [CrossRef]

35. Tian, S. Gemological Characteristics and Mineral Typomorphic Characteristics of Green Xinjiang Manasi Nephrite. Master's Thesis, Shijiazhuang University of Economics, Shijiazhuang, China, 2014.

36. Gil, G.; Barnes, J.D.; Boschi, C.; Gunia, P.; Raczynski, P.; Szakmány, G.; Bendő, Z.; Petérdi, B. Nephrite from Złoty Stok (Sudetes, SW Poland): Petrological, geochemical, and isotopic evidence for a dolomite-related origin. Can. Mineral. 2015, 53, 533-556. [CrossRef]

37. Wu, L.J. Study on Gemological and Mineralogical Characteristics and Genesis of Nephrite in Tiantai, Qiemo, Xinjiang. Master's Thesis, China University of Geosciences (Beijing), Beijing, China, 2016.

38. Gao, K. Study on the Metallogenic Mechanism of Tashisayi Nephrite from Xinjiang. Unpublished. Ph.D. Thesis, China University of Geosciences (Beijing), Beijing, China, 2018.

39. Gao, K.; Shi, G.H.; Wang, M.L.; Xie, G.; Wang, J.; Zhang, X.C.; Fang, T.; Lei, W.Y.; Liu, Y. The Tashisayi nephrite deposit from South Altyn Tagh, Xinjiang, northwest China. Geosci. Front. 2019, 10, 1597-1612. [CrossRef]

40. Han, H.W.; Zhang, C.J.; Wang, H.; Mou, L.X.; Wei, Y.; Bai, D. Metallogenic geological characteristics and prospecting prospects of nephrite deposits in the middle and western Altyn Tagh. Acta Mineral. Sin. 2011, 31, 952-953. (In Chinese)

41. Local Chronicle Compilation Committee. Ruoqiang County Local Chronicle; Chinese Literature and History Press: Ruoqiang, China, 2017. (In Chinese)

42. Yu, M. Chinese Jade Yearbook; Beijing Science Press: Beijing, China, 2017. (In Chinese)

43. Guangxi Regional Geological Survey Institute. Geological Map of the Washixia, Xinjiang, China, Scale 1: 250,000; Guangxi Regional Geological Survey Institute Press: Guilin, China, 2003. (In Chinese)

44. Xinjiang Geology and Mineral Bureau third Geology Brigade. Geological Survey of Yingelike Nephrite Mine, Ruoqiang County, Xinjiang, China; Xinjiang Geology and Mineral Bureau Third Geology Brigade Press: Kuerle, China, 2002. (In Chinese)

45. Wang, C.; Liu, L.; Xiao, P.X.; Cao, Y.T.; Yu, H.Y.; Meert, J.G.; Liang, W.T. Geochemical and geochronologic constraints for Paleozoic magmatism related to the orogenic collapse in the Qimantagh-South Altyn region, northwestern China. Lithos 2014, 202-203, 1-20. [CrossRef]

46. Wang, C.; Liu, L.; Yang, W.Q.; Zhu, X.H.; Cao, Y.T.; Kang, L.; Chen, S.F.; Li, R.S.; He, S.P. Provenance and ages of the Altyn Complex in Altyn Tagh: Implications for the early Neoproterozoic evolution of northwestern China. Precambrian Res. 2013, 230, 193-208. [CrossRef]

47. Liu, L.; Sun, Y.; Xiao, P.X.; Che, Z.C.; Luo, J.H.; Chen, D.L.; Wang, Y.; Zhang, A.D.; Chen, L.; Wang, Y.H. Discovery of ultrahigh-pressure magnesite-bearing garnet lherzolite ( $>3.8 \mathrm{GPa})$ in the Altyn Tagh, Northwest China. Chin. Sci. Bull. 2002, 47, 881-886. [CrossRef]

48. Cowgill, E.; Yin, A.; Harrison, T.M.; Wang, X.F. Reconstruction of the Altyn Tagh fault based on U-Pb geochronology: Role of back thrusts, mantle sutures, and heterogeneous crustal strength in forming the Tibetan Plateau. J. Geophys. Res. Solid Earth 2003, 108, B7. [CrossRef]

49. Zhang, J.X.; Zhang, Z.M.; Xu, Z.Q.; Yang, J.S.; Cui, J.W. Petrology and geochronology of eclogites from the western segment of the Altyn Tagh, Northwestern China. Lithos 2001, 56, 187-206. [CrossRef]

50. Mohsen, M.-D. Distant vision method. In Dictionary of Gems and Gemology, 3rd ed.; Springer: Berlin/Heidelberg, Germany, 2009; p. 271. [CrossRef]

51. Mohsen, M.-D. Hydrostatic weighing of specific gravity. In Dictionary of Gems and Gemology, 3rd ed.; Springer: Berlin/Heidelberg, Germany, 2009; p. 440. [CrossRef]

52. Meng, X.Z.; Zhao, M.F. The legal measurement unit of Vickers hardness and its conversion. J. Gems Gemmol. $2007,2,52$.

53. GB/T 14506. 30-2010. National Standards of the People's Republic of China: Methods for Chemical Analysis of Silicate Rocks-Part 30: Determination of 44 Elements; Standardization Administration of China Press: Beijing, China, 2010. (In Chinese) 
54. Clayton, R.N.; Mayeda, T.K. The use of bromine pentafluoride in the extraction of oxygen from oxides and silicates for isotopic analysis. Geochim. Cosmochim. Acta 1963, 27, 43-52. [CrossRef]

55. Liu, H.B.; Jin, G.S.; Li, J.J.; Han, J.; Zhang, J.F.; Zhang, J.; Zhong, F.W.; Guo, D.Q. Determination of stable isotope composition in uranium geological samles. World Nucl. Geosci. 2013, 3, 174-179. (In Chinese with English abstract) [CrossRef]

56. Friedman, I. Deuterium content of natural waters and other substances. Geochim. Cosmochim. Acta 1953, 4, 89-103. [CrossRef]

57. Adamo, I.; Bocchio, R. Nephrite Jade from Val Malenco, Italy: Review and Update. Gems Gemol. 2013, 49, 98-106. [CrossRef]

58. Leake, B.E.; Woolley, A.R.; Arpes, C.E.S.; Birch, W.D.; Gilbert, M.C.; Grice, J.D.; Hawthorne, F.C.; Kato, A.; Kisch, H.J.; Krivovichev, V.G.; et al. Nomenclature of Amphiboles; Report of the Subcommittee on Amphiboles of the International Mineralogical Association Commission on New Minerals and Mineral Names. Mineral. Mag. 1997, 61, 295-310. [CrossRef]

59. Morimoto, N.; Fabries, J.; Ferguson, A.K.; Ginzburg, I.V.; Ross, M.; Seifert, F.A.; Zussman, J.; Aoki, K.; Gottardi, G. Nomenclature of pyroxenes. Mineral. Petrol. 1988, 39, 55-76. [CrossRef]

60. Sun, S.-S.; McDonough, W.F. Chemical and isotopic systematics of oceanic basalts: Implications for mantle composition and processes. Geol. Soc. Spec. Publ. 1989, 42, 313-345. [CrossRef]

61. Liu, X.F.; Zhang, H.Q.; Liu, Y.; Zhang, Y.; Li, Z.J.; Zhang, J.H.; Zheng, F. Mineralogical Characteristics and Genesis of Green Nephrite from the World. Rock Miner. Anal. 2018, 23, 479-489. (In Chinese) [CrossRef]

62. Kim, S.J.; Lee, D.J.; Chang, S. A mineralogical and gemological characterization of the Korean jade from Chuncheon, Korea. J. Geol. Soc. Korea 1986, 22, 278-288. (In Korean)

63. Noh, J.H.; Yu, J.-Y.; Choi, J.B. Genesis of nephrite and associated calc-silicate minerals in Chuncheon area. J. Geol. Soc. Korea 1993, 29, 199-224. (In Korean)

64. Pei, X.X. Study on the Chuncheon Nephrite Deposit, Korea. Master's Thesis, China University of Geosciences (Beijing), Beijing, China, 2012.

65. Li, H.J.; Cai, Y.T. Study on Characteristics of Nephrite from Liyang, Jiangsu Province. J. Gems Gemmol. 2008, 10, 16-19. (In Chinese with English abstract) [CrossRef]

66. Simandl, G.J.; Riveros, C.P.; Schiarizza, P. Nephrite (Jade) Deposits, Mount Ogden Area, Central British Columbia (NTS 093N 13W). Geol. Fieldwork 2000, 1999, 339-347.

67. Mi, L.L. Green Nephrite Jade from Canada. J. Gems Gemmol. 2003, 1, 10-13, (In Chinese with English abstract). [CrossRef]

68. Zhao, Y.Y. Study of Gemological Mineralogy of Green Nephrite from New Zealand. Master's Thesis, China University of Geosciences (Beijing), Beijing, China, 2015.

69. Chen, C.X.; Chen, S.Y.; Li, G.G.; Liu, D.Y. Comparative research on gemological characteristics of Hualian nephrite from Taiwan. Acta Petrol. Mineral. 2014, S2, 35-40. (In Chinese with English abstract)

70. Yuan, M.; Wu, R.H.; Zhang, J.H. A study of gemological and color influencing ions of green nephrite from Ospinsk No. 7 mining area, Russia. Acta Petrol. Mineral. 2014, S1, 48-54. (In Chinese with English abstract)

71. Prokhor, S.A. The genesis of nephrite and emplacement of the nephrite bearing ultramafic complexes of East Sayan. Int. Geol. Rev. 1991, 33, 290-300. [CrossRef]

72. Zhang, Z.W.; Gan, F.X.; Cheng, H.S. PIXE analysis of nephrite minerals from different deposits. Nucl. Instrum. Methods Phys. Res., Sect. B 2011, 269, 460-465. [CrossRef]

73. Siqin, B.; Qian, R.; Zhou, S.J.; Gan, F.X.; Dong, M.; Hua, Y.F. Glow discharge mass spectrometry studies on nephrite minerals formed by different metallogenic mechanisms and geological environments. Int. J. Mass Spectrom. 2012, 309, 206-211. [CrossRef]

74. Siqin, B.; Qian, R.; Zhuo, S.J.; Gao, J.; Jin, J.; Wen, Z.Y. Studies of rare earth elements to distinguish nephrite samples from different deposits using direct current glow discharge mass spectrometry. J. Anal. At. Spectrom. 2014, 29, 2064-2071. [CrossRef]

75. Men, Y. The Causes and Genesis Study of Nephrite in Alamas, Yutian, Xinjiang, China. Master's Thesis, China University of Geosciences (Beijing), Beijing, China, 2014.

76. Zhou, Z.H.; Feng, J.R. A petrological and mineralogical comparison between Xinjiang nephrite and Xiuyan nephrite. Acta Petrol. Mineral. 2010, 29, 331-340. (In Chinese with English abstract)

77. Feng, X.Y.; Zhang, Y.; Lu, T.J.; Zhang, H. Characterization of Mg and Fe contents in nephrite using Raman spectroscopy. Gems Gemol. 2017, 53, 204-212. [CrossRef] 
78. Fritsch, E.; Rossman, G.R. An update on color in gems. Part 1: Introduction and colors caused by dispersed metal ions. Gems Gemol. 1987, 23, 126-139. [CrossRef]

79. Yu, H.Y.; Wang, R.C.; Guo, J.C.; Li, J.G.; Yang, X.W. Color-inducing elements and mechanisms in nephrites from Golmud, Qinghai, NW China: Insights from spectroscopic and compositional analyses. J. Mineral. Petrol. Sci. 2016, 111, 313-325. [CrossRef]

80. Guo, L.H.; Han, J.Y. The IR analyses of M1 and M3 cation occupation of Hetian jade, Manasi green jade and Xiuyanold jade. Acta Petrol. Mineral. 2002, 21, 68-71. (In Chinese)

(C) 2020 by the authors. Licensee MDPI, Basel, Switzerland. This article is an open access article distributed under the terms and conditions of the Creative Commons Attribution (CC BY) license (http://creativecommons.org/licenses/by/4.0/). 